\title{
The prediction of differential hardening behaviour of steels by multi-scale crystal plasticity modelling
}

\author{
Philip Eyckens $^{1 *}$, Hans Mulder ${ }^{2}$, Jerzy Gawad ${ }^{3,4}$, Henk Vegter ${ }^{2}$, Dirk Roose ${ }^{3}$, \\ Ton van den Boogaard ${ }^{5}$, Albert Van Bael $^{1}$, Paul Van Houtte ${ }^{1}$
}

${ }^{1}$ Department of Materials Engineering, KU Leuven, Kasteelpark Arenberg 44 - box 2450, 3001 Heverlee, Belgium

${ }^{2}$ Tata Steel Research, Development \& Technology, IJmuiden Technology Centre, PO Box 10.000, 1970 CA, IJmuiden, The Netherlands

${ }^{3}$ Department of Computer Science, KU Leuven, Celestijnenlaan 200A, 3001 Leuven, Belgium

${ }_{5}^{4}$ AGH University of Science and Technology, al. Mickiewicza 30, Krakow, 30-059, Poland.

${ }^{5}$ Nonlinear Solid Mechanics, University of Twente, PO Box 217, 7500 AE Enschede, The

Netherlands

*Corresponding author: tel (+32) 16 321305, fax (+32) 16321990 ,

Philip.Eyckens@mtm.kuleuven.be

\begin{abstract}
An essential aspect of materials modelling in the field of metal plasticity is hardening. The classical assumption of isotropic hardening in metal plasticity models is often too simplified to describe actual material behaviour. This paper focuses on the non-isotropic hardening termed differential hardening that is experimentally observed in many commercially available steel sheet materials. Crystal plasticity theory is used to study for a number of single-phase steels the differential hardening effect between uniaxial and equibiaxial loading conditions. We consider both the iso-strain assumption in polycrystal plasticity (Taylor model), and plasticity modelling with heterogeneous strain distribution across the polycrystal (Alamel model). In the latter case, the restriction of stress equilibrium along grain boundaries in selected locations throughout the microstructure dictates the nature and degree of the strain heterogeneity. In view of the potential wide applicability of the modelling approach, it has been chosen to keep the strain hardening law very simple, with only 3 fitting parameters. By doing so, differences in critical resolved shear stress (CRSS) between individual slip systems within a grain cannot be taken into account. Nevertheless, the results show significant improvement in differential hardening prediction by polycrystal plasticity modelling featuring strain heterogeneity over the microstructure, in comparison to results obtained with an iso-strain assumption. The accuracy improvement originates from the loading-dependent response in strain heterogeneity for textured steel materials. Texture evolution contributes additionally to the differential hardening effect. Steels with higher textural strength are predicted to show more differential hardening, in accordance to experiment.
\end{abstract}

Keywords: yield condition, constitutive behaviour, crystal plasticity, polycrystalline material, strain heterogeneity 


\section{Introduction}

The introduction first puts differential hardening in the general perspective of anisotropic behaviour. Next, the differential hardening phenomenon is described, in particular the deviation from isotropic hardening that is commonly found for steel grades at the start of deformation and at medium levels of deformation. Finally an overview is given of polycrystal plasticity modelling, as this approach will be followed to study the physical background of the differential hardening phenomenon.

\subsection{Modelling anisotropic constitutive behaviour}

The concept of isotropic hardening is widely used in numerical simulations of sheet metal forming processes despite the fact that even for proportional loading conditions it can only be an approximation of the actual material behaviour. Hill and Hutchinson (1992) stated that the texture development with plastic deformation causes significant changes in the successive shapes of yield loci and the material response is therefore not isotropic. This phenomenon was named differential hardening because the deviation from isotropic hardening could also be represented by a presumed dependence on the stress ratio $\sigma_{1} / \sigma_{2}$ of the strain hardening exponent in a power-law description of the stress-strain characteristic for a constant value of $\sigma_{1} / \sigma_{2}$, i.e. for proportional plane stress loading. The statement on successive yield loci was modified in Hill et al. (1994) to successive contours of equal plastic work.

The modelling approach of differential hardening by Hill builds on isotropic hardening models, in particular on a yield function and a function for the flow stress. The concept of a plastic potential for the yield criterion is introduced by von Mises (1928) and elegantly used by Hill (1948), who derived one of the most commonly known anisotropic yield criteria. The points in stress space that satisfy the yield criterion form a so called yield locus. In isotropic hardening the size of the locus in stress space is determined by the flow stress. The size of the locus grows with plastic work increments. Hill's yield criterion is brilliant in its definition but unfortunately limited in capturing anisotropic behaviour. Yield criteria that are flexible enough to capture all initial anisotropic material properties in sheet metal forming are for instance developed by Vegter (Vegter et al., 1995; Vegter and Van den Boogaard, 2006), by Banabic (Banabic et al., 2005), by Barlat (Barlat et al., 2003; Barlat et al., 2005) and by Cazacu (Cazacu and Barlat, 2004, Cazacu et al., 2006).

Yield stresses in tension and compression are usually identical, except for metals with a HCP crystal structure that demonstrate the so-called strength differential effect. Vegter's yield criterion does not impose an identical behaviour in tension and compression and is able to capture this phenomenon. Plunkett et al. (2008) have shown that Barlat's approach with linear transformations can also be applied to yield criteria that capture the strength differential effect, thereby creating the same flexibility in modelling anisotropic behaviour. When high accuracy is required in sheet metal forming simulations the full stress tensor needs to be considered, which includes components in the throughthickness direction. Barlat's Yld2004 yield criterion (Barlat et al., 2005) is defined for a three dimensional stress tensor. Vegter's yield criterion is defined for plane stress conditions. Van Riel and Van den Boogaard (2007) provided a framework for a generalization to three dimensional stress tensors in sheet metal forming.

A phenomenon that is not captured by the isotropic hardening model is the Bauschinger effect. It refers to a material property found in most polycrystalline metals. When a material is deformed, unloaded and loaded in the reverse direction, typically tension followed by compression, the tensile 
yield strength increases but the compressive yield strength is reduced. In isotropic hardening the compressive yield strength increases with an increased tensile yield strength. Prager (1949) introduced the linear kinematic rule where the yield locus is translated by the kinematic stress tensor or back stress tensor. Classical texts on the development of the back stress in cyclic loading are a.o. by Armstrong and Frederick (1966) and by Chaboche (1986). In the kinematic hardening model the size of the yield locus is unchanged. Mixed hardening models have been proposed that combine the isotropic and kinematic hardening concepts, e.g. as described in Yoshida and Uemori (2002), Wu (2002) and Chung et al. (2005).

Cyclic loading could be seen as a specific case of a strain path change and the Bauschinger effect as a specific phenomenon associated with strain path reversal. In general an initial deformation will not only influence the size and the position of the yield locus but also its shape. This modelling concept is called distortional hardening (Kurtyka and Życzkowski, 1996). It is the topic of quite a lot of recent research, a.o. by Barlat et al. (2011, 2013), Feigenbaum et al. (2012) and Freund et al. (2012). The common shape change of the yield locus with deformation is towards a sharper radius in the direction of loading and flattening in the opposite direction.

The commonality between the current concepts of isotropic, kinematic and mixed hardening is the relationship between anisotropic behaviour and flow stress in the description of yielding in continuum mechanics, given by the yield criterion:

$\phi(\boldsymbol{\sigma}-\boldsymbol{\alpha})=Y\left(\varepsilon_{e q}, \dot{\varepsilon}, T\right)$

where the yield function $\phi$ is a positive, homogeneous function of degree one and $\boldsymbol{\sigma}$ is the stress tensor in an elasto-plastic state. The back stress tensor $\boldsymbol{\alpha}$ controls the yield surface translation (zero in case of isotropic hardening), and the flow stress (yield stress) $Y$ determines the yield locus size. The scalar that results from evaluation of the yield function $\phi$ is called the equivalent stress (effective stress) $\sigma_{e q}$. Whereas plastic yielding occurs if $\sigma_{e q}=Y$, a purely elastic stress state satisfies the inequality $\sigma_{e q}<Y$. The flow stress $Y$ is a function of the equivalent plastic strain $\varepsilon_{e q}$, the strain rate $\dot{\varepsilon}$ and temperature $T$. The flow stress $Y$ is work-conjugate to the equivalent plastic strain $\varepsilon_{e q}$. Once a choice has been made for the definition of the equivalent plastic strain, the hardening $Y\left(\varepsilon_{e q}\right)$ can be derived from the dissipated plastic work measured in a reference test (usually uniaxial tensile test).

A possible interpretation of the yield criterion (1) is that the flow stress $Y$ is associated with hardening as a result of dislocation multiplication influencing critical resolved shear stresses on the slip systems, including dynamic contributions, and the yield function $\phi$ captures all anisotropic properties due to texture (and possibly texture development). Both the flow stress and the yield function can be extended with internal state variables to capture the stress and/or strain history. Yield functions with internal state variables are typically found in distortional hardening models. In principle, distortional hardening models are able to model different hardening curves in proportional, monotonic loading conditions. However, in the state-of-the art distortional hardening models (e.g. Barlat et al. (2013), Feigenbaum et al. (2012), Freund et al. (2012)), the evolution equations for the yield function, based on the internal state variables, have primarily been developed for strain path changes, not for differential hardening as defined in the next section.

\subsection{Differential hardening in steels}


Differential hardening is defined after Hill and Hutchinson (1992) as the phenomenon that the stressstrain behaviour of a metal in case of proportional, monotonic loading conditions cannot be described by a single hardening curve based on the dissipated plastic work, but rather depends on the loading condition. This phenomenon was recently observed by Mulder and Vegter (2010) for a B220H bake hardening steel as well as a DP600 dual phase steel grade.

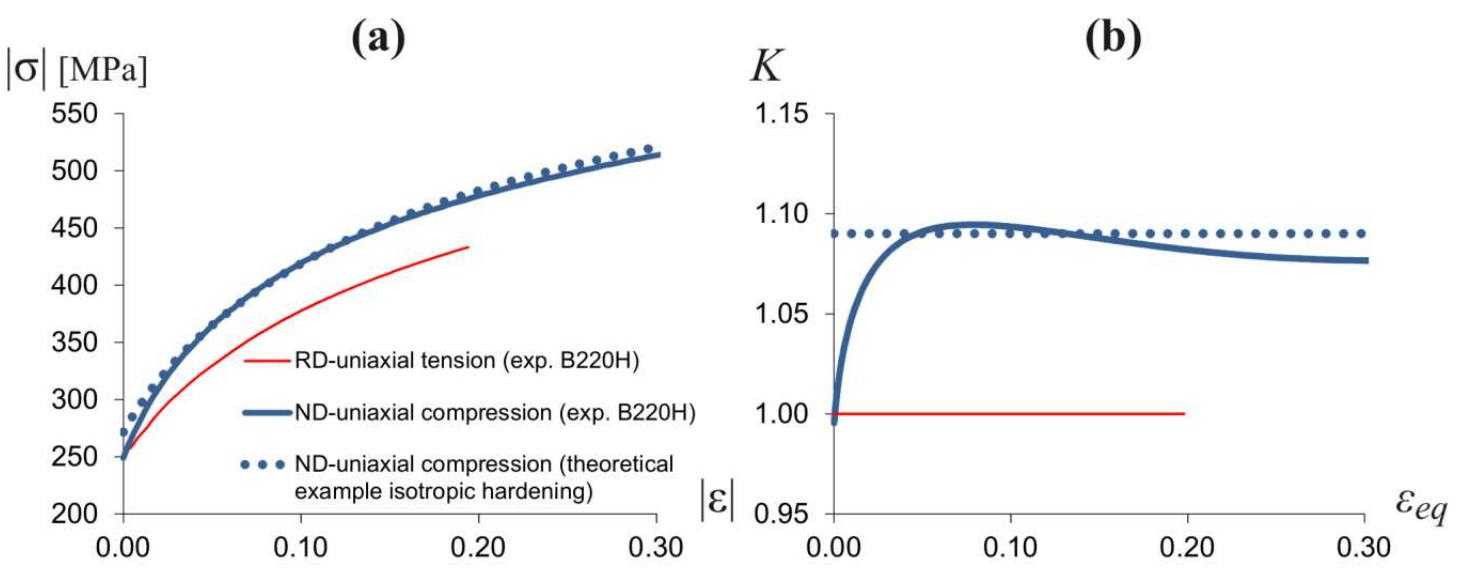

Figure 1: Differential hardening in $\mathrm{B} 220 \mathrm{H}$ bake hardening steel, after Mulder and Vegter, 2010.

Experimental data is given in full lines; the dotted line shows a theoretic example of ND-uniaxial compression behaviour under assumption of isotropic hardening (using the experimental data of RDuniaxial tension as a reference). (a) Stress-strain curves. (b) Stress factor $K$

In Figure 1.a the true stress - plastic strain curves are shown for the bake hardening steel under two loading conditions: uniaxial tension as determined in a tensile test and in-plane equibiaxial tension determined by a stack compression test along the normal direction. The uniaxial tensile test along RD usually serves as a reference, i.e. the corresponding stress-strain curve is used to specify the development of the flow stress $Y$.

In the following discussion all measures of strain refer to the plastic part of deformation (unless explicitly stated otherwise). Stress - strain curves for different loading conditions can be compared based on the dissipated plastic work:

$d W=\sigma_{e q} d \varepsilon_{e q}=\sigma_{i j} d \varepsilon_{i j}$

For a stack compression test, the right-hand side of (2) simplifies to $\sigma_{b} d \varepsilon_{b}$, with $\sigma_{b}$ the normal compressive stress and $\varepsilon_{b}$ the normal compressive strain.

Consider a particular value of equivalent strain $\varepsilon_{e q}^{*}$ and the corresponding value of plastic work $W^{*}$ (i.e. the dissipated work in the reference test at strain $\varepsilon_{e q}^{*}$ ). The equi-work stress factor $K$ for the stack compression test is then defined as:

$K=\frac{\left.\sigma_{b}\right|_{W=W^{*}}}{\left.\sigma_{e q}\right|_{W=W^{*}}}$

It follows from (2) that the corresponding strain increment $d \varepsilon_{b}$ must be a factor $1 / K$ times the equivalent strain increment from the reference stress-strain curve $d \varepsilon_{e q}$. If a metal behaves as modelled in the isotropic hardening case (i.e. equation (1) applies with back stress $\boldsymbol{\alpha}=\mathbf{0}$ ), this incremental stress factor $K$ must be constant throughout the deformation. According to (3) $K$ is a function of plastic work. This can be converted through (2) into the equivalent strain for the reference test, which is more readily recognized as a level of deformation. Figure 1.b shows $K$ against 
the equivalent strain. For the reference (uniaxial) stress $K$ is of course equal to one throughout deformation. A remarkable observation that can be made from Figure 1.b is that the stress factor $K$ for the equibiaxial stress state shows a strong development in the first $5 \%$ of equivalent strain. It is peculiar because the common referenced cause for differential hardening (e.g. Hill and Hutchinson, 1992) is texture development and it is therefore expected that $K$ is fairly constant at the start of deformation and gradually deviates at higher strains. Possible explanations for this behaviour are

- The predeformation due to temper rolling and stretching-levelling.

These operations are common to sheet metal production and are meant to improve mechanical properties, surface roughness and shape. (Peeters et al., 2001) have shown that strain path change effects can be explained from the concept of cell block boundaries formed in the predeformation. It is unlikely that a strong dislocation substructure with significant cell block boundaries that influence hardening for the next $5 \%$ is formed in the approximately $1 \%$ predeformation associated with temper rolling. It is therefore considered a less likely contribution to an explanation;

- Microplastic behaviour in the pre-yield regime.

Van Liempt and Sietsma (2014) explain the presence of microplastic behaviour as a consequence of stress gradients near grain boundaries due to anisotropic elasticity at the grain level. It is yet unclear whether this mechanism can have an impact beyond yielding, when dislocation multiplication occurs;

- Texture development and slip partitioning.

Texture development itself is a gradual process and it is generally accepted as the root cause for differential hardening when gradual changes at high deformation are observed. It is unlikely that texture development is the only explanation for the observed behaviour. Being dependent on the orientation, the partitioning of the local strain via slip over the different slip systems will generally evolve as a consequence of texture evolution;

- Development of strain heterogeneity. Individual grains will have a strain dependence on texture, microstructure and load case, which need not be identical to the macro strain. It is also known that intra-granular strain heterogeneities exist. These strain heterogeneities contribute to the anisotropic behaviour. If the strain heterogeneity changes with deformation it may contribute to differential hardening;

- Development of the critical resolved shear stress.

Hardening is known to depend on the number of dislocations. The number of dislocations is driven by micro strain and available slip systems, in other words grain (orientation) dependent. The critical resolved shear stress may therefore develop differently for each grain and contribute to developments in strain heterogeneity.

The statistical crystal plasticity model that is described in this paper is capable of modelling the last three mentioned possible causes for differential hardening. Simulations will give an indication of their individual contribution to the observed phenomenon.

\subsection{Crystal plasticity modelling}

Crystal plasticity models approximate the physical processes that govern plasticity at the smaller length scales, such as dislocation slip, twinning, phase transformations, etc. We currently limit our focus to plasticity by dislocation slip without any thermally-activated processes such as dislocation climb. For polycrystalline material, crystal plasticity models typically consider a representation or 
abstraction of the microstructure, including crystallographic texture, as well as the specific orientation and nature of the slip systems within the constituting grains. Crystal plasticity models can be classified in full-field and statistical models.

Full-field crystal plasticity models make use of a Representative Volume Element (RVE) of the microstructure, which is sufficiently large so that the average response of the RVE to a particular loading would correspond to that of the material at the macroscopic level. On the local scale, the fullfield method allows to resolve the heterogeneity of stress and strain within particular grains and across the grains. Different numerical methods have been used to solve the non-linear plasticity problem of the RVE, firstly the crystal plasticity-finite element method (CP-FEM) and more recently, the crystal plasticity-Fast Fourier method (CP-FFT). Advanced CP-FEM models, e.g. (Ma et al., 2006a, b; Roters et al., 2010), consider hundreds to thousands of elements per grain. While this clearly is a valuable tool for numerical analysis of crystal plasticity, the major downside is the large calculation time, even with state-of-the-art computing power, inhibiting practical engineering applications. This shortcoming has driven the development of the generally faster CP-FFT, see the review article of (Lebensohn et al., 2011). Yet computational costs remain several orders of magnitude larger than the class of statistical models.

Compared to full-field models, statistical crystal plasticity models make further abstraction of the microstructure, while attempting to capture the most significant effects of microstructure on the macroscopic behaviour. This generally allows them to be considerably faster than full-field methods. Advanced statistical models are seen to be accurate enough for application in engineering models of metal forming processes in which plastic anisotropy and texture evolution may be relevant factors (Van Houtte et al., 2011).

Statistical crystal plasticity models build upon the knowledge (usually acquired from measurement) of the statistical distribution of crystallographic orientations of the material under consideration. A limited but statistically representative set of crystals can be derived from the Orientation Distribution Function (ODF) (Bunge, 1982), which itself can be derived from measured pole figures of lattice orientations. The classical statistical model is the one of Taylor-Bishop-Hill (TBH, also called Taylor model) (Bishop and Hill, 1951a, b; Taylor, 1938). The number of publications on the TBH model is huge; we limit ourselves here to (Kocks, 1998). The TBH theory can be solved through linear programming (Van Houtte, 1988) or by the visco-plastic method (Asaro and Needleman, 1985). The basic TBH model assumes a homogeneous deformation of all crystals that constitute the polycrystal. In other words, the 'neighbourhood' (i.e. zone of mutual influence within the microstructure) is limited to an individual crystal. As a consequence, stress equilibrium along the boundaries of neighbouring grains is generally not satisfied at all. It is nowadays understood that this limitation is the main reason why deformation textures produced by classical TBH theory do only qualitatively agree with experimental deformation textures. A wide range of more modern statistical crystal plasticity models have been proposed which attempt to relieve this limitation, often improving quantitative deformation texture prediction. These can be roughly subdivided into long-range and short-range interaction-type statistical models, although this classification is not strict since also mixed models have been formulated, e.g. (Lee et al., 2002) and (Xie et al., 2014).

Long-range interaction models are more commonly known as Visco-Plastic Self-Consistent (VPSC) models, cf. (Lebensohn and Tomé, 1993). They consider an inclusion (typically a grain) surrounded by 
a 'homogeneous medium' that represents the polycrystal. Stress equilibrium along the modelled interface is satisfied. Many variants have been proposed, some of which are quite complex. Yet they all incorporate the long-range interaction that any individual grain experiences with its polycrystalline surrounding.

Grain interaction models on the other hand look at the short-range interaction between individual neighbouring grains and formulate certain relaxations on the strict assumption of TBH (requiring homogeneous deformation), thereby improving the stress equilibrium condition along grain boundaries. An example is the Alamel model (Van Houtte et al., 2005), which considers as basic neighbourhood a bicrystal, i.e. a grain-boundary and the two crystals at either side. This crystal plasticity model will be adopted in the present work, and its exact formulation will be detailed further on. The Alamel model has been extensively validated by deformation texture predictions for steel (Van Houtte et al., 2011; Van Houtte et al., 2005) and aluminium alloys (Delannay et al., 2009) (the latter study making use of an elastic-viscoplastic extension of Alamel). The deformation texture predictions with Alamel are seen to be of comparable quality compared to the much more calculation-intensive CPFEM model approach. More recently, the Alamel model has also been shown to produce a more realistic initial yield locus compared to Taylor (Eyckens et al., 2011). The graininteraction model of (Evers et al., 2002) includes the grain interiors (grain cores) in addition to bicrystals representing grain-boundary-affected zones. Plasticity within a bicrystal volume element is affected by the neighbouring grain through the imposed grain boundary stress equilibrium, but not within the grain core. The intra-grain crystal lattice incompatibility that is induced in this modelling approach generates Geometrically Necessary Dislocations (GNDs), which contribute to the strength. Assuming a fixed grain core volume fraction, the lattice incompatibility becomes more stringent as grain size decreases, which leads to a Hall-Petch-type relation, as illustrated by the authors for copper.

Other grain-interaction models construct a neighbourhood of more than 2 grains, such as the GIA model (Engler et al., 2005) and related RGC model (Tjahjanto et al., 2010), which feature sets of 8 hexahedral grains with 12 internal boundaries. In (Mahesh, 2010) a 'binary tree' model is proposed as a generalization of Alamel. Each node of the binary tree of certain order $i$ represents an aggregate of $2^{i}$ grains, the highest order being the modelled idealization of the entire polycrystal. Pairs of 2 nodes of the same order $i$ that form two neighbouring aggregates of crystals within the microstructure, together form a node of order $i+1$. Aggregates of order 1 are thus neighbouring grains, and they interact with each other, yet without fully complying with strain compatibility and stress equilibrium, as is done in the Alamel model. Their interaction effects are nonetheless integrated in the higher-order nodes. Compared to Alamel, this model captures a more flexible heterogeneity of deformation across the polycrystal. The model is applied to various macroscopic loading conditions, yet its capability to predict differential hardening cannot readily be assessed from (Mahesh, 2010), as different sets of hardening parameters are assigned for different loading conditions. Another extension of Alamel towards a larger interacting neighbourhood is the stack model (Arul Kumar et al., 2011), replacing the 2-grain stack of Alamel by an N-grain stack with parallel interfaces. It is a promising approach to represent 'banded' microstructures (shear bands, twinning bands, flattened grains, etc.) without excessive calculation requirements in comparison to the Taylor model.

\section{Theory}


This section revisits the polycrystalline plasticity models that will be used for differential hardening prediction further on. In the first paragraph, the single crystal plasticity model is elaborated. Both the Taylor and Alamel homogenization assumptions are discussed next. They are enriched with a macroscopic flow stress formulation, or alternatively with an evolution equation for the Critical Resolved Shear Stress (CRSS) in the individual constituting grains (termed microscopic hardening).

Each combination of homogenization and hardening models will lead to a yield criterion such as (1). For sake of simplicity, strain-rate sensitivity and temperature-dependent behaviour will be neglected in the analysis. In addition, the development of back stress will not be considered, given that the application of differential hardening excludes the occurrence of Bauschinger effect. The yield criterion that is to be obtained, is thus of the form:

$\phi(\boldsymbol{\sigma}, \mathbf{x})=Y\left(\varepsilon_{e q}, \mathbf{x}\right)$

In this equation $\mathbf{x}$ represents the internal state variables of the crystal plasticity model. Note that all strains are plastic unless stated otherwise.

\subsection{Crystal plasticity model}

Suppose a crystal or grain is subjected to an instantaneous deformation, described in general by the local velocity gradient $\mathbf{l}$. This velocity gradient, imposed onto the crystal, is composed of a symmetric part, i.e. the local strain rate $\mathbf{d}$, and an anti-symmetric part, called the local spin $\mathbf{w}$. Any non-zero local strain rate $\mathbf{d} \neq \mathbf{0}$ needs to be realized through plastic deformation, which is carried by dislocation slip on a number of slip systems.

Dislocation slip on a slip system $(s)$ is described by a simple shear on the slip plane with unit normal vector $\widehat{\mathbf{n}}^{(s)}$. Let $\hat{\mathbf{b}}^{(s)}$ be the normalized burgers vector, which defines the shear direction. The velocity gradient tensor that describes a unit of slip of $(s)$ is given by the tensorial product $\hat{\mathbf{b}}^{(s)} \otimes \widehat{\mathbf{n}}^{(s)}$. The Schmid tensor $\mathbf{M}^{(s)}$ is defined as:

$\mathbf{M}^{(s)}=\left(\hat{\mathbf{b}}^{(s)} \otimes \widehat{\mathbf{n}}^{(s)}+\widehat{\mathbf{n}}^{(s)} \otimes \hat{\mathbf{b}}^{(s)}\right) / 2$

The amount of slip per unit time is given by the shear rate ('slip rate') $\dot{\gamma}^{(s)}$. The simultaneous slipping of a number of slip systems realizes the strain rate inside the grain $\mathbf{d}$ :

$\mathbf{d}=\sum_{(s)} \mathbf{M}^{(s)} \dot{\gamma}^{(s)}$

The slip systems for which $\dot{\gamma}^{(s)} \neq 0$ are called the active slip systems. For ferritic (BCC) steels, 24 slip systems are assumed to be potentially active: $12\{110\}<111>$ and $12\{112\}<111>$ slip systems. The imposed strain rate tensor has however only 5 independent components, considering it is symmetric and traceless (due to plastic incompressibility). For such a large number of slip systems in comparison to the independent components of $\mathbf{d}$, an infinite number of solutions for all $\dot{\gamma}^{(s)}$ in (6) exist. An additional assumption is required to restrict the number of possible solutions. Taylor proposed to retain the solution(s) with minimal dissipation of plastic work (Taylor, 1938). This energetic criterion will be formulated mathematically further on in section 2.2, as it is different between the adopted homogenization schemes (FC Taylor or Alamel). In each scheme however, it involves the rate of plastic work in the crystal per unit volume, also called plastic power $p$, which is in general given by:

$p=\sum_{(s)} \tau_{c}^{(s)}\left|\dot{\gamma}^{(s)}\right|$ 
Here, the critical resolved shear stress (CRSS) of a slip system $\tau_{c}^{(s)}$ is the scalar measure of stress that is work-conjugate to the respective slip rate $\dot{\gamma}^{(s)}$. In principal, $\tau_{c}^{(s)}$ may be different for the two considered slip system families due to the different atomic configuration in $\{110\}$ and $\{112\}$ planes, and may also be different between forward and negative slip on $\{112\}$ slip planes (stress differential effect). Moreover, the development of an anisotropic cell substructure with ongoing plastic deformation will generally lead to different values of CRSS between the individual slip systems, and also between the two slip directions for a particular slip system (development of back stress). In this paper however, all these differences are neglected and it is assumed that, at least inside each grain, the CRSS is the same for all slip systems; it is noted as $\tau_{c}$. Hence (7) reduces to

$p=\tau_{c} \sum_{(s)}\left|\dot{\gamma}^{(s)}\right|=\tau_{c} \dot{\Gamma}$

in which $\dot{\Gamma}$ is the total slip rate inside the grain.

Suppose the active slip systems and their slip rates are known by solving (6) together with the energetic criterion. According to (6), geometric compatibility in terms of the local strain rate is accomplished. In order to satisfy the local geometric compatibility completely, i.e. in terms of the local velocity gradient, also the local spin $\mathbf{w}$ needs to be considered. In general, a rate of rotation of the crystal lattice of the grain (called the crystal lattice spin $\mathbf{w}^{\mathbf{L}}$ ) is required to realize the imposed $\mathbf{w}$ :

$\mathbf{w}=\mathbf{w}^{\mathbf{L}}+\sum_{(s)} \frac{\left(\hat{\mathbf{b}}^{(s)} \otimes \widehat{\mathbf{n}}^{(s)}-\widehat{\mathbf{n}}^{(s)} \otimes \hat{\mathbf{b}}^{(s)}\right)}{2} \dot{\gamma}^{(s)}$

The second term on the right-hand side is called the plastic spin. It is the anti-symmetric part of the velocity gradient realized by slip. Note that unlike $\mathbf{w}^{\mathbf{L}}$, the plastic spin does not cause lattice rotation. Equation (9) forms the basis to calculate $\mathbf{w}^{\mathbf{L}}$ after the slips have been calculated, resulting in a grain lattice rotation after a finite deformation.

\subsection{From crystal to polycrystal: homogenization assumptions}

Consider now the deformation of a polycrystalline aggregate, consisting of a statistically significant number of grains (a few thousand) derived from the (measured) Orientation Distribution Function $f^{c}(g)$, in which the superscript $c$ refers to the crystallographic texture. A macroscopic velocity gradient $\mathbf{L}$ is imposed onto the polycrystal. This velocity gradient tensor, which is 'imposed on the polycrystal', comprises in general a symmetric part (D) and anti-symmetric part (W), so that $\mathbf{L}=\mathbf{D}+\mathbf{W}$. Different homogenization assumptions may be made that relate these quantities to the respective local (grain) quantities. In the following, we focus only on the symmetric part $\mathbf{D}$. The antisymmetric part $\mathbf{W}$ is paramount in the calculation of the deformation texture of the polycrystal. As this topic falls out of the scope of the present paper, the interested reader is referred to (Van Houtte, 1988; Van Houtte et al., 2005).

The energetic criterion for the polycrystalline material postulates a minimal plastic power over the whole polycrystal:

$\bar{P} \rightarrow$ Min.

Note that, according to the equivalence of Taylor and Bishop-Hill theorems, the minimal internal plastic dissipation in the polycrystal relates to the macroscopic stress $\boldsymbol{\sigma}$ and macroscopic strain rate D according to: 
In terms of the local deformation inside the constituting grains, the formulation of the energetic criterion will depend on the homogenization scheme, as elaborated next.

\subsubsection{Taylor model}

The Taylor model (Taylor, 1938) neglects any strain partitioning over the polycrystal, i.e. the local velocity gradient equals the macroscopic one, so also

$\mathbf{d}=\mathbf{D}$

Given that the left-hand side of (6) is fully prescribed through (12), it is sufficient to require the energetic criterion (10) for each of the constituting crystals separately. Using (8), the energetic criterion becomes for a particular grain:

$p=\tau_{c} \dot{\Gamma} \rightarrow$ Min.

As $\tau_{c}$ can be regarded as a state variable of the whole grain, it is in fact sufficient to minimize $\dot{\Gamma}=p / \tau_{c}$. Furthermore, it is for numerical reasons preferred to minimize a dimensionless quantity. By convention, the von Mises equivalent strain rate of the polycrystal $D_{v M}=\sqrt{2 / 3}\|\mathbf{D}\|$ is used for this purpose. The dimensionless Taylor factor $M$ of the grain is thus defined and introduced in the solution of the energetic minimization problem:

$M \stackrel{\text { def }}{=} \frac{\dot{\Gamma}}{D_{v M}} \rightarrow$ Min

The minimization problem of (14) with the linear constraints of (6) and (12) can be efficiently solved using linear programming (Van Houtte, 1988).

The value of the obtained Taylor factor $M$ of the grain corresponding to the solution, depends on the instantaneous orientation of the grain $g$ and on the applied strain rate mode $\mathbf{A} \stackrel{\text { def }}{=} \mathbf{D} /\|\mathbf{D}\|=$ $\sqrt{2 / 3} \mathbf{D} / D_{v M}$. This is noted in short as $M(\mathbf{A}, g)$. It can be remarked that the Taylor factor $M$ does not depend on the magnitude of the applied strain rate, which is a consequence of the assumed strainrate insensitivity.

Consequently, the volume-average Taylor factor (usually called 'average Taylor factor') $\bar{M}$ is a function of both $\mathbf{A}$ and the (instantaneous) crystallographic texture of the polycrystal $f^{c}(g)$ :

$\bar{M}=\bar{M}\left(\mathbf{A}, f^{c}(g)\right)$

\subsubsection{Alamel model}

The Alamel model (Van Houtte et al., 2005) considers a neighbourhood of two grains (orientations $g 1$ and $g 2$ ) with an associated planar grain boundary with orientation $g^{B}$. The polycrystal constitutes a large number (a few thousands) of such clusters, with initial grain orientations assigned from the crystallographic texture in the form of a known Orientation Distribution Function (ODF) $f^{c}(g)$, and initial grain boundary orientations assigned from the Grain Boundary Orientation Distribution Function $f^{m}\left(g^{B}\right)$; the superscript $m$ in this notation stands for the morphologic texture. 
On top of the macroscopic deformation $\mathbf{D}$, simple shearing along the grain boundary is allowed in the local strain description. This "deformation relaxation" (in comparison to Taylor) is equal in magnitude in both grains but opposite in sign. The local strain rates are mathematically expressed as:

$\mathbf{d}^{g 1}=\mathbf{D}+\left(\widetilde{\mathbf{M}}_{1} \dot{\gamma}_{R L X 1}+\widetilde{\mathbf{M}}_{2} \dot{\gamma}_{R L X 2}\right)$

$\mathbf{d}^{g 2}=\mathbf{D}-\left(\widetilde{\mathbf{M}}_{1} \dot{\gamma}_{R L X 1}+\widetilde{\mathbf{M}}_{2} \dot{\gamma}_{R L X 2}\right)$

Here, $\widetilde{\mathbf{M}}_{\mathbf{1}}$ and $\widetilde{\mathbf{M}}_{\mathbf{2}}$ are pseudo-Schmid tensors for the simple shear deformation along the grain boundary plane in two perpendicular directions, while $\dot{\gamma}_{R L X 1}$ and $\dot{\gamma}_{R L X 2}$ are the corresponding slip rates (also called relaxation rates).

It can be seen in (16) that the average of the local deformations equals the macroscopic deformation D. Because of this coupling between $\mathbf{d}^{g 1}$ and $\mathbf{d}^{g 2}$, the expression of the energetic criterion (10) in terms of local deformations is to be done for the cluster as a whole, i.e.:

$p^{g 1}+p^{g 2}=\tau_{c}^{g 1} \dot{\Gamma}^{g 1}+\tau_{c}^{g 2} \dot{\Gamma}^{g 2} \rightarrow$ Min.

Note that the CRSS in both grains are not necessarily equal to each other (cf. section 2.3.2).

The energetic minimization problem is in practice formulated as a weighted sum of the Taylor factors of both grains:

$\tau_{c}^{g 1} M^{g 1}+\tau_{c}^{g 2} M^{g 2} \rightarrow$ Min.

The minimization problem (18) with the linear constraints of (6) and (16) can also be solved through linear programming (Van Houtte et al., 2005), the two relaxation rates in (16) being formally treated as two additional slip systems without any associated slip resistance (zero CRSS) (Van Houtte et al., 2005).

In the Alamel model, the obtained Taylor factor $M$ of any grain depends on the orientation of both grains in the cluster, and additionally on the grain boundary orientation, i.e. $M\left(\mathbf{A}, g 1, g 2, g^{G B}\right)$. The average Taylor factor $\bar{M}$ of the polycrystal is thus a function of:

$\bar{M}=\bar{M}\left(\mathbf{A}, f^{c}(g), f^{m}\left(g^{G B}\right)\right)$

A measure of average strain heterogeneity across the polycrystal $\kappa$ can be generally defined as:

$\kappa=\frac{\overline{\|\mathbf{d}-\mathbf{D}\|}}{\|\mathbf{D}\|}$

By using (16), and considering that $\left\|\widetilde{\mathrm{M}}_{i}\right\|=1 / \sqrt{2}$ and that the pseudo-Schmid tensors are by definition orthogonal to each other $\left(\widetilde{\mathbf{M}}_{1}: \widetilde{\mathbf{M}}_{2}=0\right), \kappa$ equals for the Alamel model

$\kappa=\frac{\sqrt{\left(\dot{\gamma}_{R L X 1}\right)^{2}+\left(\dot{\gamma}_{R L X 2}\right)^{2}}}{\sqrt{2}\|\mathbf{D}\|}$

\subsection{Strain hardening}


In the crystal plasticity framework, the most natural definition of a work-conjugate equivalent stress and equivalent strain is based on the rate of plastic work as given by (8) for an individual grain.

An obvious choice for the equivalent strain of the polycrystal, is the volume-average accumulated slip $\bar{\Gamma}:$

$\varepsilon_{e q}=\bar{\Gamma}$

in which the accumulated slip within a grain $\Gamma$ is obtained through time integration of the grain slip rate $\dot{\Gamma}$ :

$\Gamma=\int \dot{\Gamma} d t$

Differences in CRSS between the constituting grains can be either neglected, or else explicitly included in the strain hardening definition. The former choice, which corresponds with a prescribed evolution equation of the (macroscopic) flow stress $Y\left(\varepsilon_{e q}\right)$, is termed macroscopic strain hardening, and is elaborated next. Another choice would be to prescribe the CRSS on the level of the individual grain, which will be denoted by microscopic strain hardening (cf. section 2.3.2). The macro- and micro- strain hardening assumptions lead to different flow stresses that are work-conjugate to the equivalent strain (22).

\subsubsection{Macroscopic definition of strain hardening}

Consider a polycrystal for which it is assumed that the CRSS (noted as $\tau_{c}$ ) is identical for each constituting grain at any given time. From (8), the volume-average rate of plastic work per unit volume is then given by:

$\bar{P}=\tau_{c} \bar{\Gamma}$

Equation (24) states in fact that $\tau_{c}$ is an equivalent stress that is work-conjugate to the equivalent strain of (22). Using the superscript ' $M$ ' referring to the macroscopic strain definition, it is noted as:

$Y^{\mathrm{M}}=\tau_{c}$

From (24)-(25) and the definition of the Taylor factor of a grain in (14), it follows that

$\bar{P}=Y^{\mathrm{M}} \bar{M} D_{v M}$

The flow stress $Y^{\mathrm{M}}$ is a function of the average (accumulated) slip across the polycrystal $\bar{\Gamma}$. In this paper, the Swift hardening law is adopted:

$Y^{\mathrm{M}}(\bar{\Gamma})=Y^{0}\left(1+\frac{\bar{\Gamma}}{\bar{\Gamma}^{0}}\right)^{N}$

with $Y^{0}, \bar{\Gamma}^{0}$ and $N$ the 3 macroscopic Swift hardening parameters: $Y^{0}$ is the initial value of the flow stress (yield stress), $\bar{\Gamma}^{0}$ the initial average slip, and $N$ the strain hardening coefficient of the macroscopic Swift law. 
Combining (11) with (26), the following relation between the macroscopic stress tensor $\boldsymbol{\sigma}$ and the flow stress is obtained:

$\boldsymbol{\sigma}: \mathbf{A}=\sqrt{2 / 3} \bar{M} Y^{\mathrm{M}}$

Equation (28) states that the macroscopic stress is the result of (i) a strain hardening contribution $Y^{\mathrm{M}}(\bar{\Gamma})$ and (ii) a contribution from the material state, which is expressed by $\bar{M}(\mathbf{A}, \mathbf{x})$. The material state $\mathbf{X}$ is essentially the crystallographic texture for the Taylor model - cf. (15), while it contains in addition the morphological texture for the Alamel model - cf. (19). So-called textural hardening is captured by the dependency of $\bar{M}$ on the instantaneous texture $f(g)$, which evolves during deformation through crystal lattice rotation of the grains constituting the polycrystal.

Comparing (28) to (4) the yield function of the polycrystal plasticity model can be identified as:

$\phi(\boldsymbol{\sigma}, \mathbf{x})=\sqrt{3 / 2} \frac{\boldsymbol{\sigma}: \mathbf{A}}{\bar{M}(\mathbf{A}, \mathbf{x})}$

Indeed, the right-hand side of (28) is a positive homogeneous function of degree one in $\boldsymbol{\sigma}$.

\subsubsection{Microscopic definition of strain hardening}

A microscopic strain hardening relation postulates the evolution of the CRSS inside a grain $\left(\tau_{c}\right)$ as a function of the accumulated slip of that grain $(\Gamma)$. Similarly to (27), we adopt a Swift-type hardening equation:

$\tau_{c}(\Gamma)=\tau_{c}^{0}\left(1+\frac{\Gamma}{\Gamma^{0}}\right)^{n}$

in which $\tau_{c}^{0}, \Gamma^{0}$ and $n$ are the 3 microscopic Swift hardening parameters: $\tau_{c}^{0}$ is the initial value of the CRSS, $\Gamma^{0}$ the initial slip, and $n$ the strain hardening coefficient of the microscopic Swift law.

Given that the slip rate and the accumulated slip are generally different for grains with different crystal orientations, $\tau_{c}$ is no longer unique for the polycrystal at a particular deformation stage (except for the undeformed stage since $\tau_{c}(\Gamma=0)=\tau_{c}^{0}$ for all grains). The volume-average rate of plastic work is therefore given by:

$\bar{P}=\overline{\tau_{c} \dot{\Gamma}}$

Note that the flow stress of the polycrystal for the microscopic hardening assumption, denoted $Y^{\mu}$, is not prescribed (unlike $Y^{\mathrm{M}}$; cf. (27)). Being formally defined in $\bar{P} \stackrel{\text { def }}{=} Y^{\mu} \bar{\Gamma}$, it can however be readily calculated by the multi-scale model using (31):

$Y^{\mu}=\overline{\tau_{c} \dot{\Gamma}} / \bar{\Gamma}$

The flow stress of the microstructural hardening approach $Y^{\mu}$ is dependent on the state of material, in particular on the CRSS $\tau_{c}$ in all the constituting grains $g$ of the polycrystal : $Y^{\mu}\left(\varepsilon_{e q}, \tau_{c}(g)\right)$.

\subsection{Resulting yield criteria}

The form of the yield criterion resulting from the polycrystalline plasticity modelling (4) depends on two distinct choices in modelling.

Firstly, it has to be chosen whether the yield locus $\phi$ is prescribed to be a function of the current material state $\mathbf{x}$ or not. If texture evolution is neglected, the initial texture fully determines the yield 
locus: $\phi(\boldsymbol{\sigma})$. If texture evolution is on the other hand considered in the model, the resulting yield locus changes shape with deformation due to evolution of the texture $f: \phi(\sigma, f)$. The instantaneous texture $f$ (a state variable) comprises the crystallographic texture $f^{c}$ for Taylor, and additionally the morphological texture $f^{m}$ for Alamel.

Secondly, the flow stress $Y$ may either be directly prescribed directly as a function of equivalent strain (macroscopic hardening: $Y^{(M a)}\left(\varepsilon_{e q}\right)$, or alternatively it follows in the microscopic hardening approach from a prescribed evolution of CRSS: $Y^{\mu}\left(\varepsilon_{e q}, \tau_{c}\right)$. Here, $\tau_{c}$, the instantaneous values of CRSS for all constituting grains is also a state variable as it depends on the deformation history.

To summarize, the following forms of yield criteria are possible in the current framework:

- $\phi(\boldsymbol{\sigma})=Y^{\mathrm{M}}\left(\varepsilon_{e q}\right)$ :

A purely isotropic hardening model (no texture evolution)

- $\phi(\sigma, f)=Y^{\mathrm{M}}\left(\varepsilon_{e q}\right)$ :

A macroscopic hardening model combined with distortional hardening due to texture evolution

- $\phi(\boldsymbol{\sigma})=Y^{\mu}\left(\varepsilon_{e q}, \tau_{c}\right)$ :

A microscopic hardening model without texture evolution

- $\phi(\sigma, f)=Y^{\mu}\left(\varepsilon_{e q}, \tau_{c}\right)$ :

A microscopic hardening model with distortional hardening due to texture evolution

\section{Results}

\subsection{Experimental observations}

\subsubsection{Material description}

Differential hardening is usually ignored in numerical simulations because it is assumed to be relevant only at high strains. The observations by Mulder and Vegter (2010) for a B220H bake hardening steel as well as a DP600 dual phase steel grade however show a significant shift in stress factor between uniaxial and equibiaxial loading conditions within the first $5 \%$ of deformation. To reduce the possible metallurgical explanations as much as possible, three single-phase, low carbon steel grades were selected, as shown in Table 1: two interstitial-free steel grades (one batch annealed and one continuously annealed, rephosphorized grade), as well as one continuously annealed high strength, low alloy grade.

\begin{tabular}{|c|ccc|}
\cline { 2 - 4 } \multicolumn{1}{c|}{} & IF-1 & IF-2 & HSLA \\
\hline type & $\begin{array}{c}\text { batch annealed } \\
\text { interstitial-free }\end{array}$ & $\begin{array}{c}\text { rephosphorized } \\
\text { interstitial-free }\end{array}$ & $\begin{array}{c}\text { High Strength } \\
\text { Low Alloy }\end{array}$ \\
$\mathrm{t}[\mathrm{mm}]$ & $\mathrm{DC06}$ & $\mathrm{H} 220 \mathrm{YD}$ & H340LAD \\
$\sigma_{\mathrm{Y}(0.2 \%)}[\mathrm{MPa}]$ & 0.7 & 1.0 & 1.0 \\
& 139 & 233 & 358 (lower);
\end{tabular}




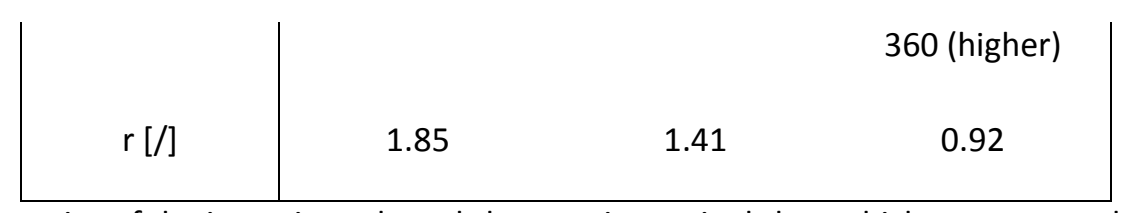

Table 1: Properties of the investigated steel sheets; $t$ is nominal sheet thickness, $\sigma_{Y(0.2 \%)}$ the $0.2 \%$ nonproportionality limit of tensile test along $0^{\circ}$ to rolling direction RD, and $r$ the Lankford coefficient of tensile test along $0^{\circ}$ to $\mathrm{RD}$.

The crystallographic textures were obtained by four incomplete XRD pole figure measurements $(\{110\},\{200\},\{112\}$ and $\{103\})$. IF- 1 and IF- 2 were measured at $50 \%, 25 \%$ and at $0 \%$ of sheet thickness. The texture gradient across thickness was seen to be very weak. HSLA was only measured at $50 \%$ of thickness. Figure 2 shows the resulting Orientation Distribution Functions (ODF) (average from the measurements over sheet thickness for IF-1 and IF-2). Also given is the textural strength of the ODF, as provided by the Texture Index (T.I.) (Bunge, 1982). IF-1 and IF-2 can be seen to have comparable textural strength and qualitatively comparable texture, comprising of a sharp $\mathrm{Y}$-fibre and partial $\alpha$-fibre. Still, some differences can be observed, notably that IF-2 has a somewhat stronger partial $\alpha$-fibre, including the rotated Cube texture component. HSLA also shows a $\gamma$-and $\alpha$-fibre texture, but the overall texture is much weaker compared to IF-1 and IF-2, which is reflected by the relatively small T.I. of 2.1 .

All 3 materials in this study possess initially a grain shape that is near-equiaxed. A random Grain Boundary Orientation Distribution Function $f^{m}$, reflecting an equiaxed initial grain shape, is assumed in all the Alamel calculations.

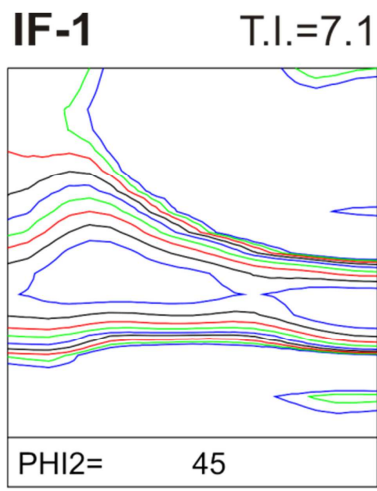

$\begin{array}{lllll}.70 & 1.00 & 1.40 & 2.00 & 2.80 \\ 4.00 & 5.60 & 8.00 & 11.0 & 16.0\end{array}$
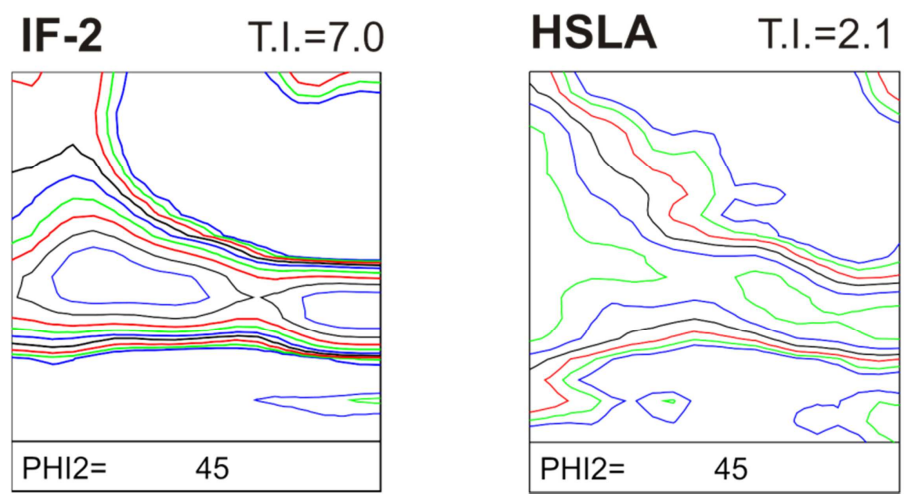
Figure 2: $\varphi_{2}=45^{\circ}$-ODF sections (horizontal axis: $\varphi_{1}$-angle in range $0^{\circ}-90^{\circ}$ from left to right; vertical axis: $\Phi$-angle in range $0^{\circ}-90^{\circ}$ from top to bottom) and Texture Index (T.I.).

\subsubsection{Differential hardening}

For a proper physical understanding of differential hardening at the start of deformation it is important to start from a yield stress that is the true onset of plastic deformation. The $0.2 \%$ nonproportional extension for the proof strength is in this respect an arbitrary definition without a physical basis. Van Liempt and Sietsma (2014) have shown that a clearly identifiable initial yield point $Y^{0}$ can be observed in a graph of the hardening rate as a function of the flow stress, which provides a well-defined and physically based yield criterion. Figure 3 shows a graph of the hardening rate $\Theta=d \sigma / d \varepsilon$ versus the flow stress $\sigma$ for the average of three uniaxial tensile tests for IF-1.

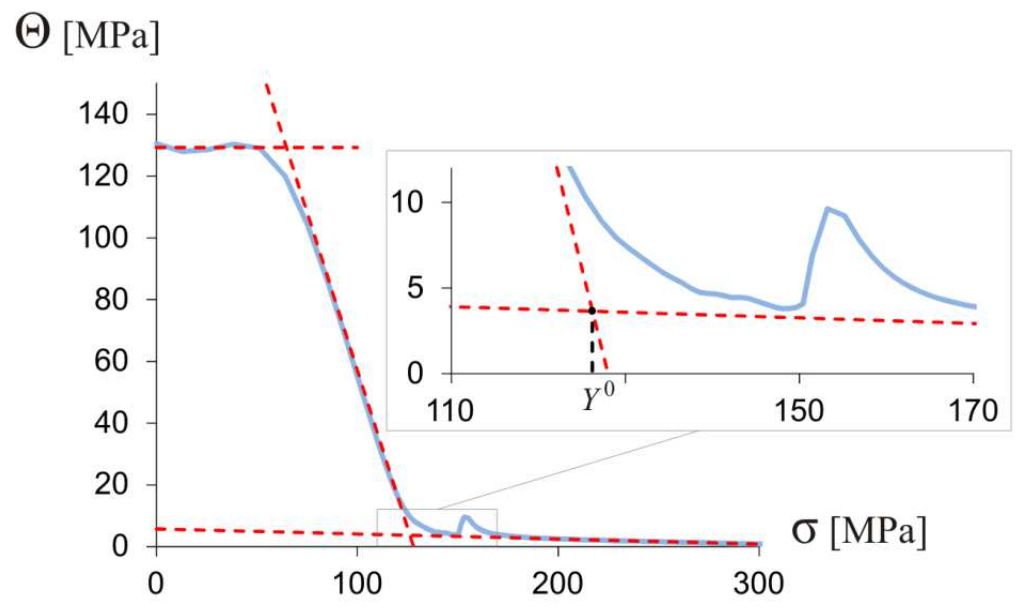

Figure 3: Kocks-Mecking plot for IF-1 (solid line; average of three uniaxial tensile tests). Young's modulus $E$ and the initial flow stress $Y^{0}$ (derived from this plot) are indicated.

The jump in hardening rate at a flow stress of 150-155 MPa is due to a strain rate change in the tensile test that is described in the Euronorm (EN 10002). To compensate for this dynamic effect, which also influences the stress factor, the flow stress is divided into two components according to a classical abstraction by Bailey and Hirsch (1960) and by Mecking and Kocks (1981):

$Y=Y^{\text {stat }}(\rho)+Y^{d y n}(\dot{\varepsilon}, T)$.

This classical abstraction assumes that at a given dislocation density $\rho$ the flow stress $Y$ depends on strain rate and temperature (combined concept of thermal activation), but the dislocation density does not. The term $Y^{\text {stat }}$ represents the static contribution of the dislocation substructure that develops with deformation, while the term $Y^{d y n}$ represents the influence of thermally activated dislocation glide. A sound physically based description of this term is due to Krabiell and Dahl (1981):

$Y^{d y n}(\dot{\varepsilon}, T)=\sigma_{0}^{*}\left(1+\frac{k T}{\Delta G_{0}} \ln \frac{\dot{\varepsilon}}{\dot{\varepsilon}_{0}}\right)^{m}$.

In this equation $k=8.61710^{-5} \mathrm{eV} / \mathrm{K}$ is Boltzmann's constant and $\Delta G_{0}=0.8 \mathrm{eV}$ the maximum Gibbs free energy barrier. The value for $\sigma_{0}^{*}$ is fitted to experimental results using different strain rates, $\dot{\varepsilon}_{0}=10^{8} \mathrm{~s}^{-1}$ is a fixed maximum for the strain rate, and $m$ is set to 2.2. This equation is used to subtract the 
influence of strain rate and temperature, in accordance with the assumptions made for the theoretical approach in the previous chapter. The effectiveness of this subtraction is clearly visible in Figure 4 as the jump in the hardening rate has been completely eliminated.

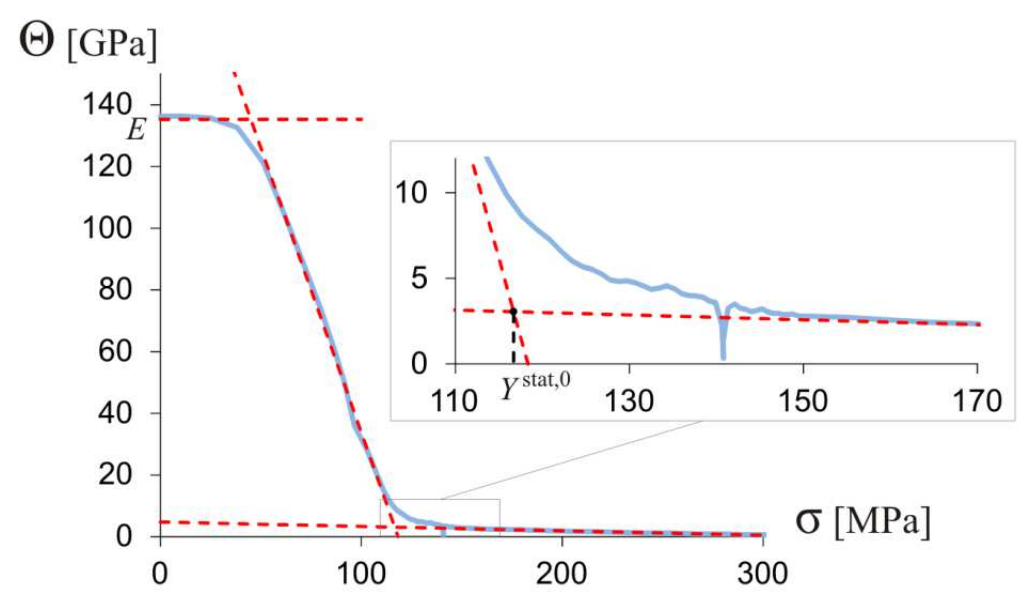

Figure 4: Kocks-Mecking plot for IF-1 after correction for strain rate (solid line). Young's modulus $E$ and the initial static part of flow stress $Y^{\text {stat, } 0}$ (derived from this plot) are indicated.

The above procedure has also been used on two types of equibiaxial stress-strain curves for IF-1: the stack compression test, described by An and Vegter (2005) and the biaxial tensile test using a cruciform specimen, described by Kuwabara et al. (1998). The three corrected stress-strain curves are plotted in Figure 5 up to 0.01 (total) elongation, showing the elastic stage and beginning of elasto-plastic stage.

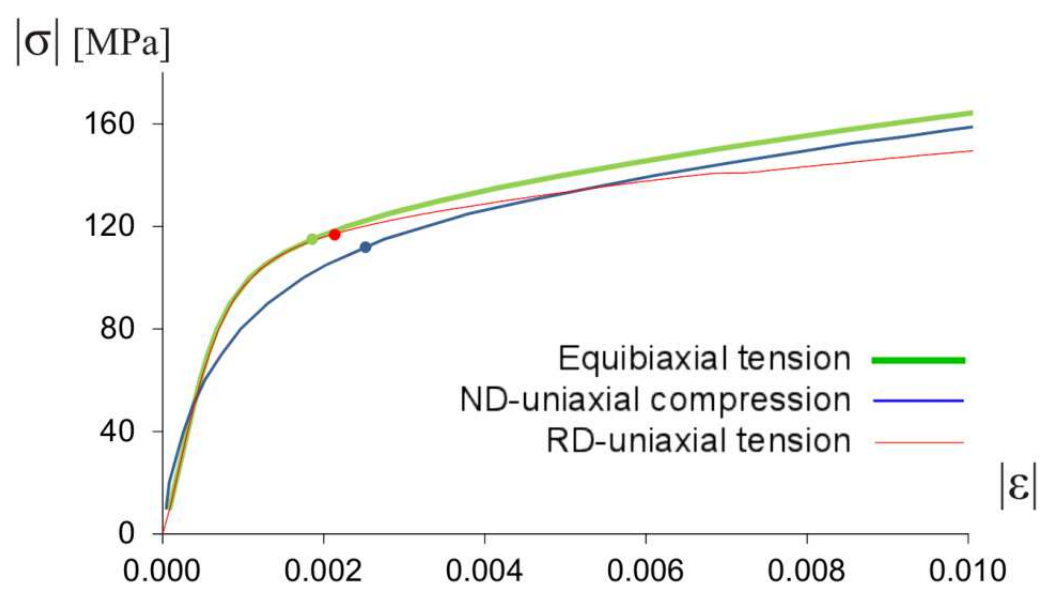

Figure 5: Stress-strain curves for three tests on IF-1 at the start of deformation. Note that the horizontal axis shows the total strain, comprised of elastic and plastic parts. For each curve, the point of initial plastic flow is marked by a dot.

In the paper by Van Liempt and Sietsma (2014) the pre-yield strain is subdivided in a linear elastic strain $\left(\varepsilon_{\text {lin }}=\sigma / E\right)$ and a non-linear anelastic strain. They show that this anelastic behaviour is due to mechanisms in the current dislocation structure. Dislocation multiplication marks the start of plasticity. The corresponding points of initial flow are indicated in Figure 5 by dots. To obtain the plastic strain the linear elastic strain as well as the non-linear anelastic (pre-yield) strain is subtracted 
from the total strain. When this rule is applied the stress-strain curves in Figure 6 result. Note that the HSLA material exhibits a mild form of yield point elongation at the onset of plasticity.
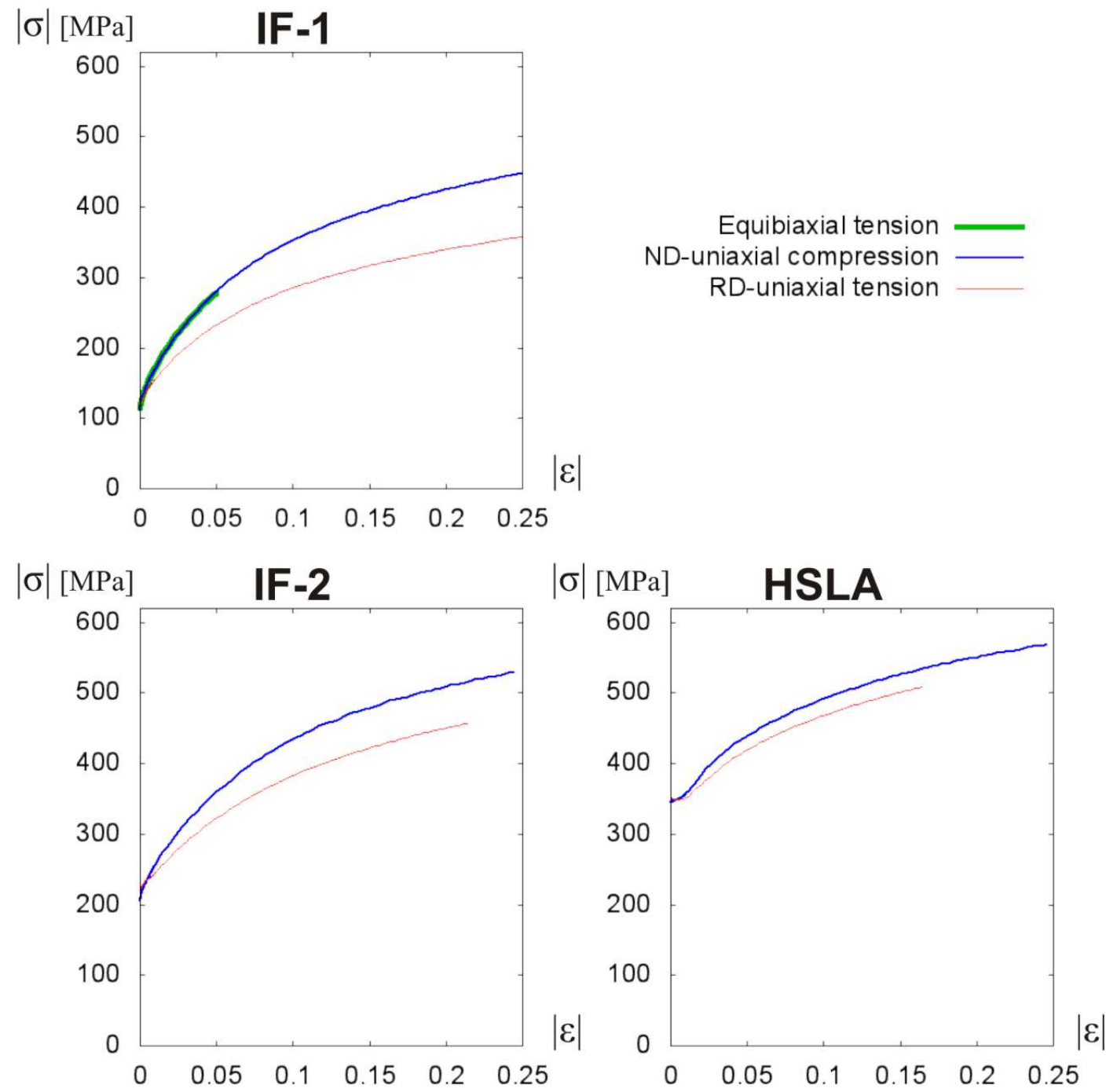

Figure 6: Experimental stress-strain curves, each curve averaged for three individual experiments. The absolute value of the true tensile (resp. compressive) stress is plotted against the absolute value of the true tensile (resp. compressive) strain.

In case of isotropic hardening the stress factor $K$ between equibiaxial stress and uniaxial stress for identical levels of plastic work, as in (2), is constant. Figure 7 shows the experimental development of $K$ where the plastic work on the horizontal axis is calculated back to the equivalent strain of the uniaxial tensile test, i.e. the area under the uniaxial tensile test is the reference. 


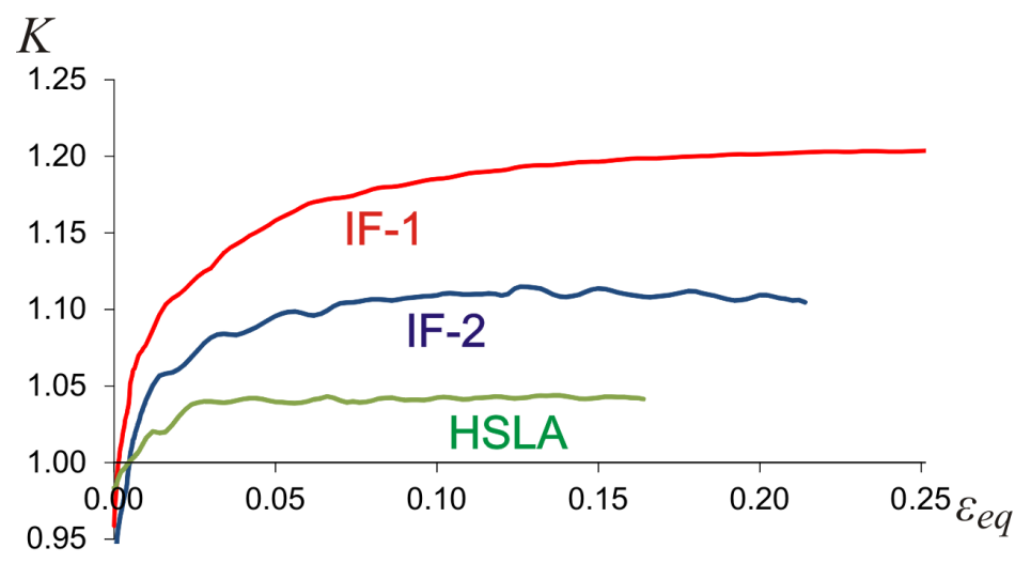

Figure 7: Stress factor $K$ (defined in (3)) from experimental tests, as a function of the tensile strain in the RD-tensile test.

Figure 7 clearly shows the same tendency for all three materials. Equibiaxial yielding occurs at almost the same level as for uniaxial tension. Within the first $5-10 \%$ deformation the ratio develops towards a more or less constant level.

\subsection{Crystal plasticity predictions of the differential hardening effect}

In this section, the effect of the various multi-scale modelling approaches introduced in section 2 is investigated in detail for the IF-2 sheet. The macro- and microscopic hardening model frameworks are combined with a polycrystal homogenization model (Taylor or Alamel), in which textural evolution during deformation is either enabled or not. The combination of microscopic hardening without texture evolution is however not considered in the following analysis, meaning that a total of 6 multi-scale model approaches will be considered. For each of these models, the hardening parameters are obtained by least-square fitting to the stress-strain curve of a tensile test along RD. Table 2 lists the sets of identified hardening parameters for the various models. More details on the hardening parameter identification are provided in appendix $A$. The adopted algorithm to realize stress-based loading conditions (e.g. uniaxial tension and compression), is elaborated in appendix B.

As can be seen in Figure 8 from the uniaxial tension curves (from experiment and various models), the quality of the hardening parameter fit is good and very comparable for all models. The predictions of the 6 identified multi-scale models for ND-compressive loading cases are also given in Figure 8. 


\begin{tabular}{|c|c|ccc|ccc|}
\cline { 2 - 7 } & $\begin{array}{c}\text { Texture } \\
\text { evolution }\end{array}$ & \multicolumn{3}{|c|}{ Macro strain hardening } & \multicolumn{4}{|c|}{ Micro strain hardening } \\
& $Y^{0}$ & $\bar{\Gamma}^{0}$ & $N$ & $\tau_{c}^{0}$ & $\Gamma^{0}$ & $n$ \\
{$[\mathrm{MPa}]$} & {$[/]$} & {$[/]$} & {$[\mathrm{MPa}]$} & {$[/]$} & {$[/]$} \\
\hline \multirow{2}{*}{ Taylor } & no & 74.5 & 0.0429 & 0.280 & - & - & - \\
& yes & 74.3 & 0.0387 & 0.265 & 74.3 & 0.0392 & 0.265 \\
\hline \multirow{2}{*}{ Alamel } & no & 80.6 & 0.0399 & 0.280 & - & - & - \\
& yes & 80.4 & 0.0381 & 0.276 & 80.4 & 0.0384 & 0.276 \\
\hline
\end{tabular}

Table 2: Hardening parameters of IF-2 for various models. 
Taylor
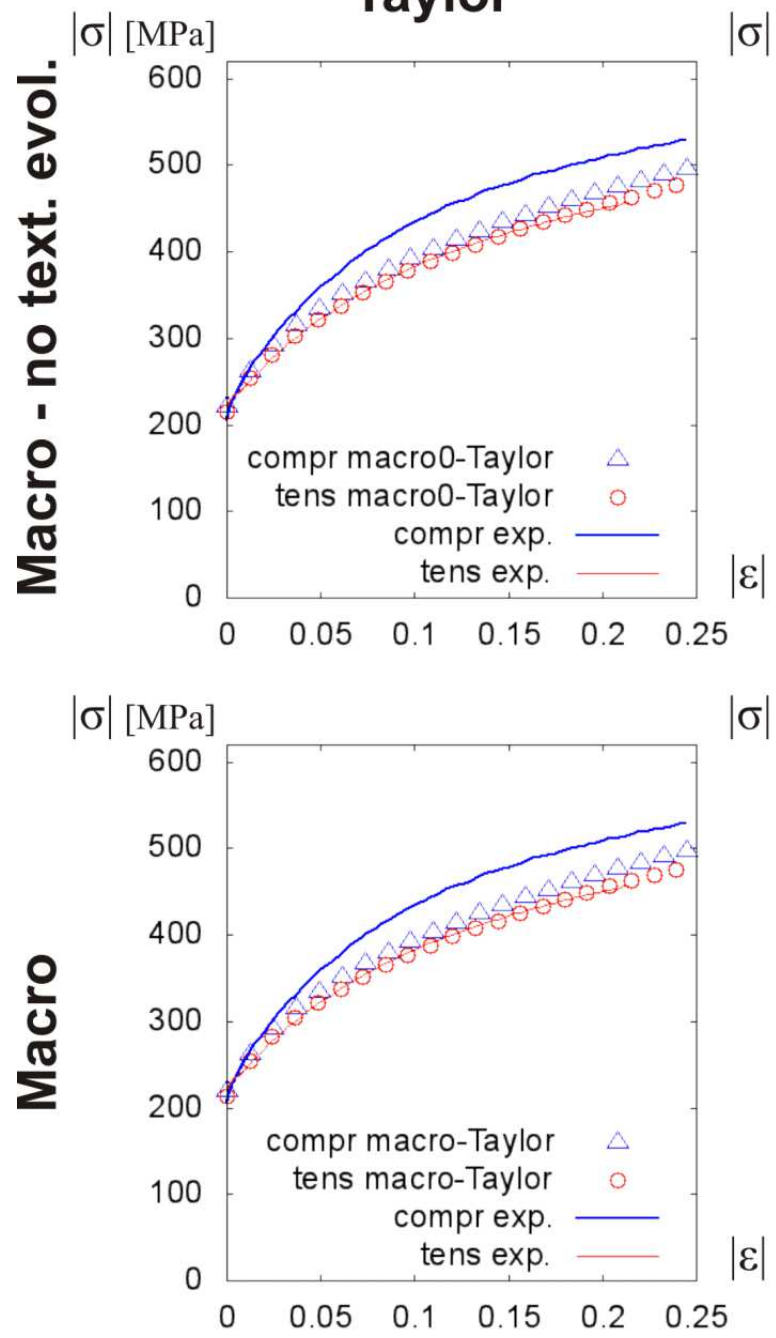

Alamel

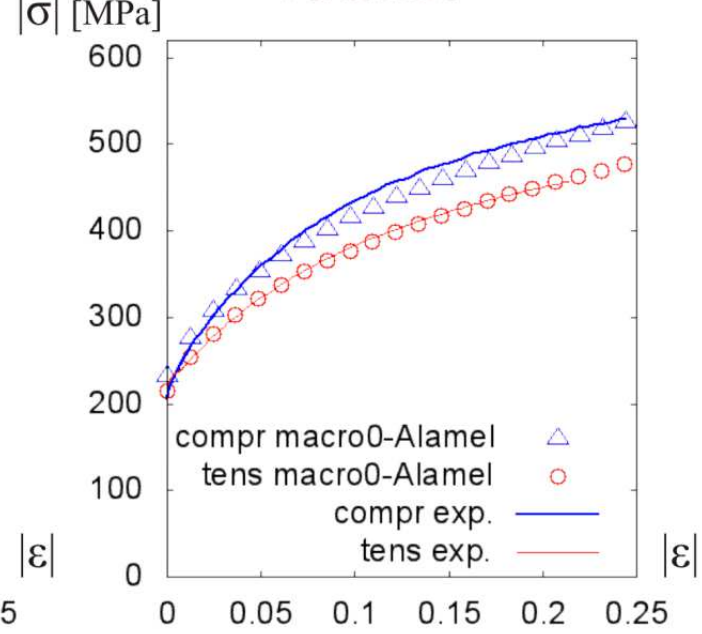

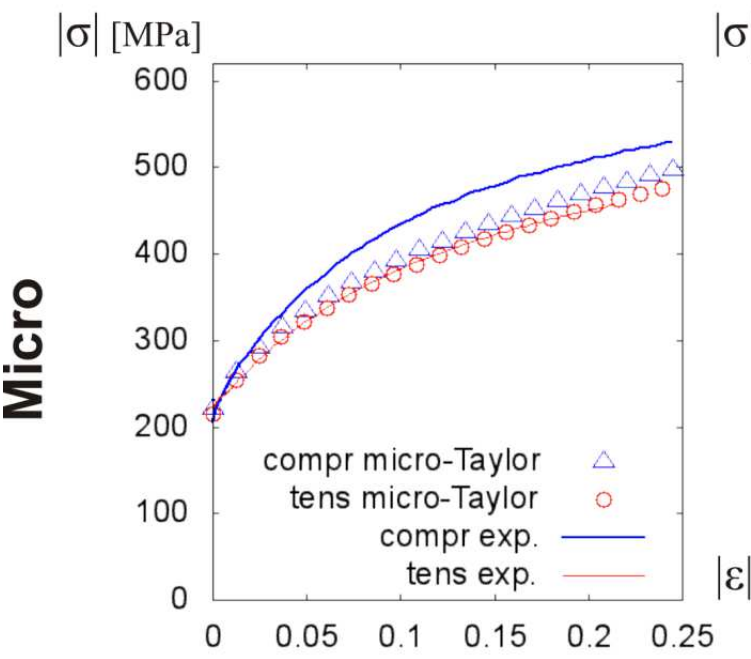

$|\sigma|$ [MPa]
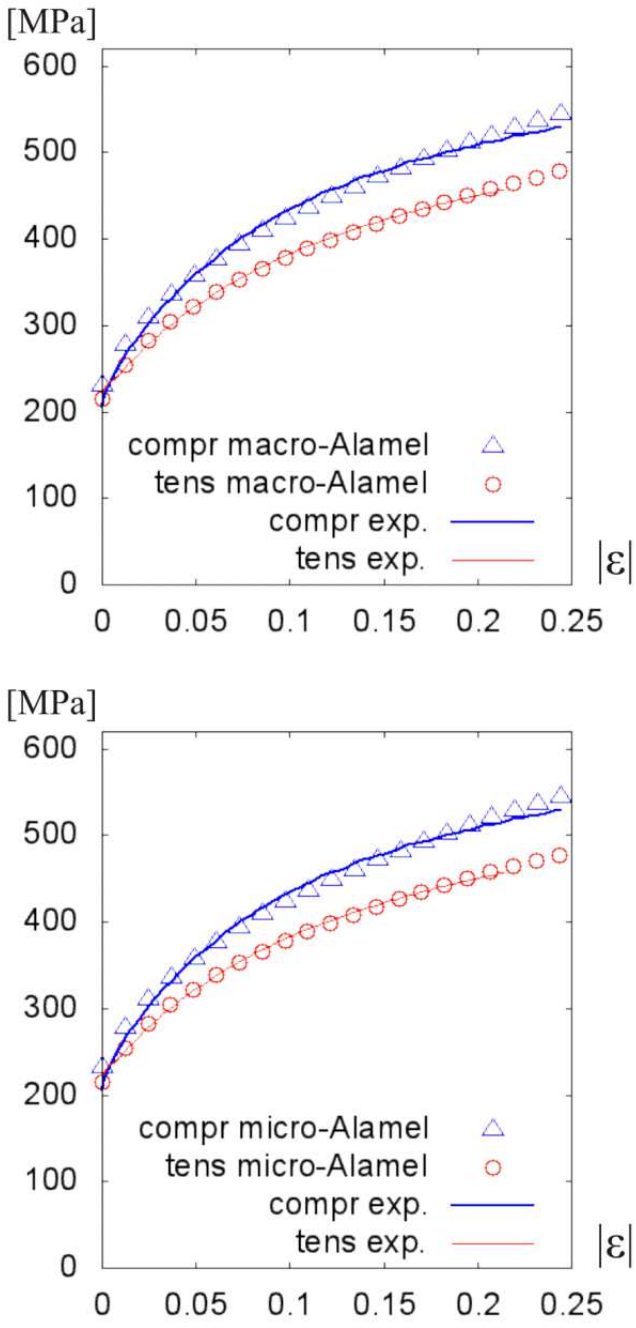

Figure 8: Stress-strain curve predictions by various models compared to the experimental curves for IF-2. 'tens': RD-tensile test; 'compr': ND-compression test. 'Macro' (resp. 'Micro'): macroscopic (resp. 
microscopic) definition of strain hardening. The absolute value of the true tensile (resp. compressive) stress is plotted against the absolute value of the true tensile (resp. compressive) strain.

The most striking difference in predictive quality between the various models presented in Figure 8 , is between Taylor and Alamel. The Alamel curves are in each case closer to experiment than Taylor, both for macroscopic hardening (without and with texture evolution) and microscopic hardening (with texture evolution). This will be investigated in more detail further on.

Considering the results for the macroscopic hardening model, it can be seen that the consideration of texture evolution in the multi-scale model clearly improves the model prediction for Alamel homogenization, while for Taylor such improvement is absent. It is known that texture prediction of the Alamel model is different from Taylor (usually better), although the differences are relatively small at the low deformation levels under consideration here (Xie et al., 2013). These results suggest that the Alamel homogenization scheme includes some physics relevant to stress-strain behaviour that is neglected by the Taylor homogenization. Comparing macro- and micro-hardening formulations, hardly any difference in the predicted ND-compression stress curves can be observed, for both the Taylor and Alamel homogenization models. Note also that the set of hardening parameters is very similar (cf. Table 2). It appears that the macroscopic strain hardening definition is a reasonable approximation of the prescription of the critical resolved shear stress as function of accumulated slip in a grain (microscopic strain hardening), at least regarding monotonic flow stress predictions.

The significant difference in the hardening predictions between Taylor and Alamel observed in Figure 8 , can be partially attributed to the different initial yield loci produced by these models. Figure 9 shows the section of the initial yield locus in which the total tensile stress along RD and TD is plotted along the horizontal and vertical axis, respectively. The yield point at RD-uniaxial tension is used as reference for normalization. The yield point for ND-compression is not present in this section directly. It can however be represented by the equibiaxial yield point, given that both stress states differ only in hydrostatic stress, while the plasticity models are insensitive to the hydrostatic stress (incompressible plasticity). It can be seen that the Alamel yield locus is significantly larger in the biaxial straining region of stress space compared to Taylor. So it can be concluded that the larger difference between compression and tensile curves in Figure 8 for Alamel compared to Taylor, can be partly attributed to the initial yield locus shape. 


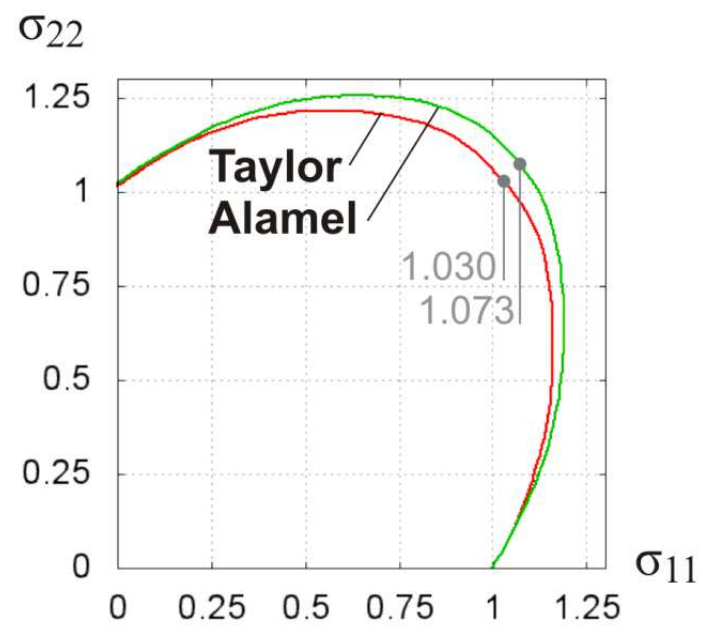

Figure 9: Initial IF-2 yield loci section (normalized by the RD uniaxial yield stress) for Taylor and Alamel. Horizontal axis: tensile stress along RD-direction; vertical axis: tensile stress along TDdirection. The ordinates of equibiaxial yield points are printed in grey. The initial yield loci are independent of the assumed hardening model.

Normalized initial yield loci are unaffected by the hardening model framework. So the differences in yield loci in Figure 9 must be a direct consequence of the different homogenization assumptions between Alamel and Taylor. The nearest-neighbour grain interaction considered by Alamel (and absent in the Taylor model), introduces a strain rate heterogeneity across the polycrystal at each stage of deformation. The degree of strain rate heterogeneity is reflected by the quantity $\kappa$ that has been introduced in equation (20). Figure 10 presents the evolution of $\kappa$ for compression and tensile predictions of the Alamel model (with either macro- or micro- strain hardening).

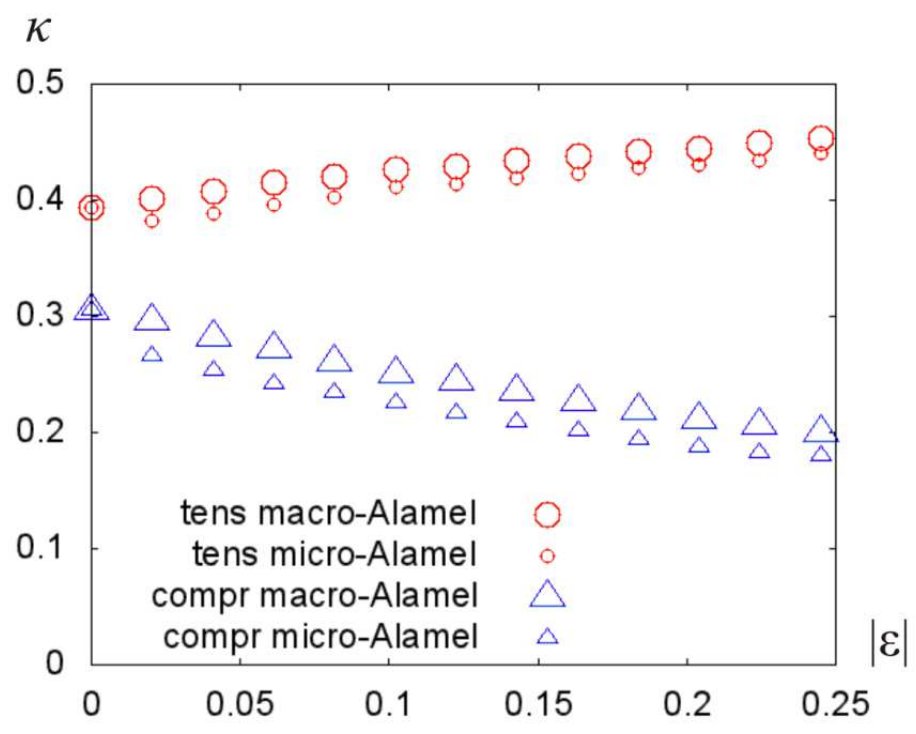


Figure 10: The strain rate heterogeneity across the polycrystal $\kappa$ (defined in (20)) in macro- and micro-Alamel models for IF-2, as a function of the absolute value of the true tensile/compression strain. 'tens': RD-tensile test; 'compr': ND-compression test. The Taylor model does not consider strain rate heterogeneity, therefore for Taylor $\kappa=0$.

It can be seen from Figure 10 that strain rate heterogeneity is not much influenced by the hardening framework (macro or micro). It depends however significantly on the loading. This difference can be attributed to the texture. Recalling that this IF- 2 has a strong $\gamma$-fibre texture, i.e. many crystals have $\{111\}$ planes closely aligned with the sheet plane, the grain-to-grain interaction is seen to be notably different for a uniaxial in-plane loading (tens) than for a uniaxial loading perpendicular to the sheet plane (compr). The relatively low strain rate heterogeneity for compressive loading (compared to tension, cf. Figure 10) means that the Alamel solution of crystallographic slip resembles more closely the Taylor solution, and so also the plastic work dissipation will be more comparable between both models. On the other hand, the uniaxial tension (tens) features significantly more strain rate heterogeneity, so the plastic work according to Alamel will be significantly lower than for Taylor (work dissipation of Taylor is an upper bound to the work dissipation of Alamel; cf. eq. (14) in comparison to eq. (18)). The reduced plastic work for Alamel translates into a lower flow stress of tensile loading (with respect to compression). Since the tensile loading is used to fit the hardening parameters, the net result is a relative increase in prediction for ND-compression, as is indeed observed in Figure 8.

For IF-2, Figure 11 compares the evolution of the stress factor $K$, defined in (3), from all these models with the experiment. Note that the initial stress factor (undeformed state, i.e. $W=0$ ), is directly related to the initial yield locus (cf. grey numbers in Figure 9).

In the absence of texture evolution, $K$ remains constant with straining, reflecting a purely isotropic hardening model without a distortional hardening component. The Alamel predictions with texture evolution show a steady increase of the stress factor $K$ during deformation, as is also observed experimentally. However, the drastic increase in $K$ that is experimentally observed for smaller strains $\left(0<\varepsilon_{e q}<0.05\right)$, is not captured by any of the model predictions.

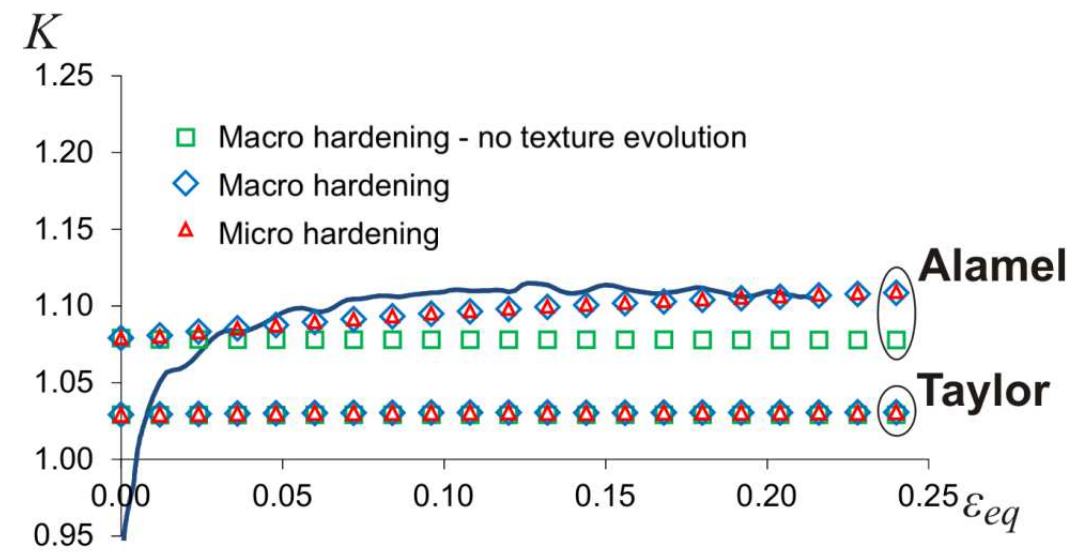

Figure 11: Stress factor $K$ (defined in (3) from experimental tests (solid line) and various models (symbols) for IF-2, as a function of the tensile strain in the RD-tensile test. 


\subsection{Differential hardening effect for steels with different texture}

This section adopts the micro-Alamel model with texture evolution (i.e. the best multi-scale model of previous section together with macro-Alamel model). The three materials described in section 3.1.1 will be investigated. Again, the hardening parameters are obtained by least-square fitting to the stress-strain curve of a uniaxial tensile test along RD (see Appendices A-B for details on the procedure). Table 3 provides the sets of identified hardening parameters.

Figure 12 presents for this multi-scale model the stress-strain curves of the uniaxial tension identification test, and in addition the predictions for ND-compressive loading. The results are reasonably satisfying. IF-1 and IF- 2 both have a relatively sharp texture (high texture index). The significant differential hardening is captured very well for IF-2, yet for IF-1 the ND-compressive loading stress is still under predicted to some extent. Possible reasons for this discrepancy are mentioned in the discussion section that follows. Note that the considerably higher flow stresses of IF-2 compared to IF-1 are automatically taken into account by the hardening law identification procedure. Predictions for the HSLA steel, with small differential hardening effect, are also clearly captured in the modelling approach. It is reasonable to assume that it is primarily the texture input to the multi-scale model (in this case a weak texture) which lies at the origin of the small differential hardening prediction, in close correspondence to experiment.

\begin{tabular}{|c|ccc|}
\cline { 2 - 4 } \multicolumn{1}{c|}{} & \multicolumn{3}{|c|}{ Micro hardening } \\
\cline { 2 - 4 } & $\tau_{c}^{0}$ & $\Gamma^{0}$ & $n$ \\
{$[\mathrm{MPa}]$} & {$[/]$} & {$[/]$} \\
\hline IF-1 & 42.1 & 0.0092 & 0.271 \\
\hline IF-2 & 80.4 & 0.0384 & 0.276 \\
\hline HSLA & 117.9 & 0.0337 & 0.185 \\
\hline
\end{tabular}

Table 3: Micro-Alamel hardening parameters of the 3 grades. 

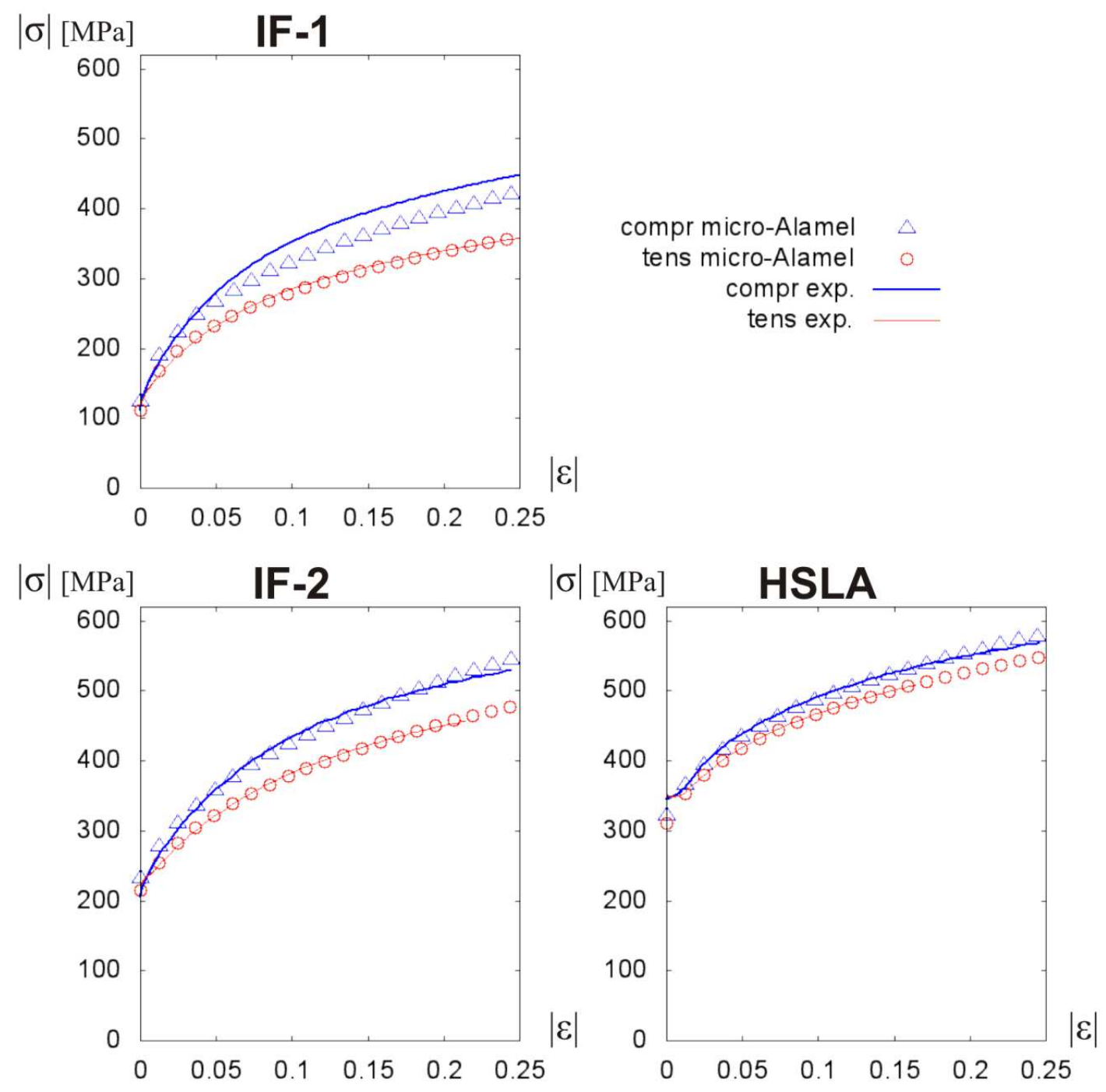

Figure 12: Stress-strain curve predictions by micro-Alamel model compared to the experimental curves. 'tens': RD-tensile test; 'compr': ND-compression test. The absolute value of the true tensile (resp. compressive) stress is plotted against the absolute value of the true tensile (resp. compressive) strain.

A final model validation is made in terms of the stress factor $K$ defined by (3) and presented in Figure 13. The model predicts a gradual increase in stress factor that corresponds roughly to the experimental trend at the higher end of strain levels. Also the material trend in magnitude of $K$ in the higher strain regime $\left(\varepsilon_{e q}>0.05\right)$ corresponds between experiment and prediction, i.e. for IF-1 it is the highest, for IF-2 intermediate, and for HSLA it is the lowest. However, the sharp increase in $K$ that is experimentally found for IF- 1 and IF- 2 in the smaller strain regime $\left(0<\varepsilon_{e q}<0.05\right)$, is not reproduced by the multi-scale modelling approach. 


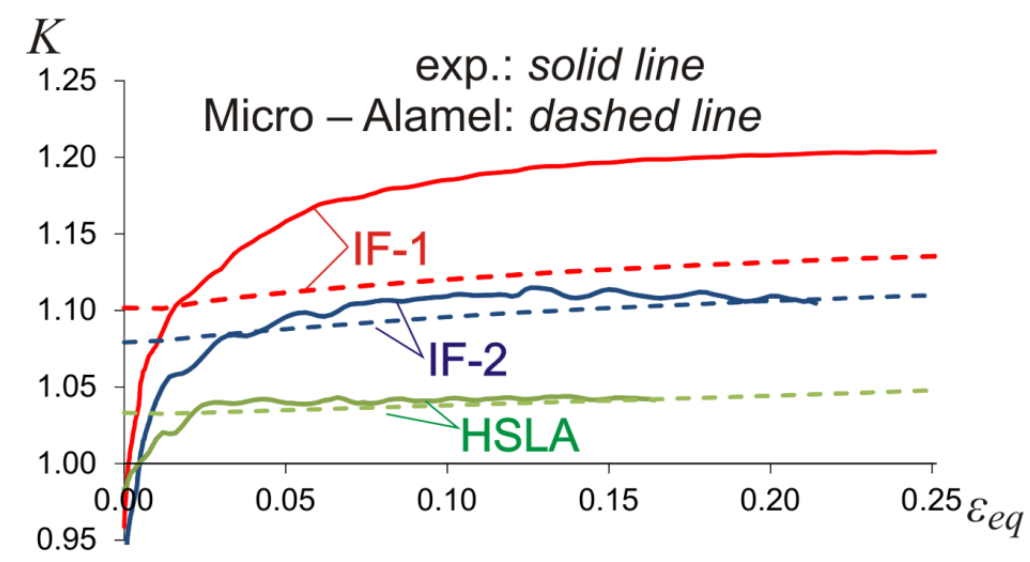

Figure 13: Stress factor $K$ (defined in (3) from experimental tests (solid lines) and micro-Alamel model (dashed lines), as a function of the tensile strain in the RD-tensile test.

\section{Discussion}

Of all crystal plasticity models studied in this work, the most accurate predictions of differential hardening was obtained with the grain-interaction Alamel model featuring texture evolution. Regarding the description of strain hardening, a basic assumption has been that the CRSS of all slip systems within any grain are equal to one another. Hardening has been either described on the microscopic scale (i.e. the CRSS is a function of the accumulated slip within that grain), or alternatively, a flow stress has been prescribed directly on the macroscopic scale, i.e. as a function of the homogenized accumulated slip of the polycrystal. The first option (microscopic strain hardening) reflects more directly the physical process of strain hardening through dislocation multiplication, whereas the second choice (macroscopic strain hardening definition) has been shown to be a very good approximation in terms of differential hardening prediction.

Macroscopic flow stress predictions of this kind of crystal plasticity model approach are remarkably accurate (especially for IF-2 and HSLA), in particular when considering the limited input that is required per material, i.e. the initial texture and the 3 hardening parameters of the adopted Swift law. The hardening parameters are identified in a straightforward manner by fitting to a single macroscopic stress-strain curve, in casu the uniaxial tension along RD. The accuracy of the obtained results is also remarkable because the Alamel crystal plasticity model has not been developed for the present application of differential hardening, but rather for the prediction of deformation texture, which is not very sensitive to the assumed strain hardening model (Van Houtte et al., 2005).

It is obvious from Figure 8 and Figure 11 that the deformation relaxation assumption in statistical crystal plasticity models is essential for accurate hardening predictions. The Alamel model, which accounts to a certain degree for strain heterogeneity, is much more accurate than the iso-strain Taylor model. It is believed that the discrepancy between experimental and predicted stress may be further reduced by refining the crystal plasticity modelling: full field crystal plasticity simulations can potentially give additional accuracy, as strain heterogeneity may be more realistically incorporated. For instance, intra-granular strain heterogeneities and long-range grain interactions are neglected in Alamel but may be readily incorporated in full field crystal plasticity simulations. 
Another opportunity for model improvement is to take the development of anisotropic dislocation substructures in account. For $\gamma$-fibred, low-carbon steel sheet it has been observed in TEM micrographs that the substructural development depends on the loading condition (Fernandes and Schmitt, 1983). Notably between in-plane uniaxial and equibiaxial loading conditions, the substructure as observable in the sheet plane section, appears very different: planar cell block boundaries (also called dense dislocation sheets or geometrically necessary boundaries (GNBs)) oriented along the mostly slipped planes are seen in uniaxial tension, while under equibiaxial tension cell boundaries without any apparent preferred orientation are the dominant feature of the observed substructure. In particular the build-up of GNBs from the onset of plastic deformation leads to increasingly intragranular heterogeneity of lattice orientation and associated slip, which has a notable effect on the flow stress (Hansen and Juul Jensen, 2011). Single-crystal plasticity experiments also indicate that the development of equiaxed substructure is accompanied with (a) a relative high slip activity on $\{112\}$ planes in comparison with $\{110\}$ planes and (b) a high initial strain hardening rate (Uenishi et al., 2009). These observations are linked in this study to the differential hardening observed between equibiaxial and uniaxial tension for $\gamma$-fibred polycrystalline IF steel. Refinement in crystal plasticity modelling that takes substructural features into account can therefore potentially lead to increased accuracy in flow stress predictions. It is hypothesized that the origin of current under prediction of the biaxial stress for IF-1 (cf. Figure 12) could lay in particular substructural developments of this alloy. Further experimental investigation is needed to study this further.

The addition of texture evolution in the Alamel model results in the prediction of differential hardening as a process of gradual change, typically observed at higher deformation. The remarkable steep increase of the stress factor between equibiaxial and uniaxial tension in the first few percent of deformation is not explained by the current crystal plasticity simulations, cf. Figure 13. A possible explanation can be found in the absence of elastic deformation in the crystal plasticity models. The entire polycrystal is assumed to be plastically deforming and the flow stress is established as a volume-average. The active slip systems, and thereby the contribution of the individual grains to the flow stress, are determined by the grain orientation. Initially all grains start with the same critical resolved shear stress and the initial flow stress therefore depends on the texture of the undeformed sheet and the strain distribution (strain heterogeneity). The strain distribution is texture and load case dependent. This reasoning assumes that there is one initial point (at zero plastic strain) where the stress exceeds the critical resolved shear stress on all slip systems. Van Liempt and Sietsma (2014) have developed an alternative reasoning: yielding is identified as the transition in dislocation behaviour from non-linear elasticity and reversible glide (without essential changes in the dislocation structure) to dislocation multiplication in the post-yield stage. The transition can be observed in a Kocks-Mecking plot, as is also shown in this paper. Pre-yield deformation is the combination of the elastic deformation of the crystal lattice and reversible dislocation glide. Due to the (strong) anisotropic elasticity at the crystal orientation level, strain discontinuities will develop at the grain boundaries. These strain discontinuities are the result of misorientations between neighbouring grains and they cause grain boundary stresses. When the pre-yield stress, including the grain boundary stress contribution, exceeds the stress at which Frank Read sources become activated, the dislocation structure starts to change and yielding occurs. This alternative argumentation starts from inter-grain misorientations. These misorientations may be more isotropic than the texture is, and consequently yielding in equibiaxial stress should be almost equal to yielding in uniaxial tension, as is indeed observed experimentally (Figure 6). This hypothesis can in principle be investigated with 
detailed full field elasto-plastic crystal plasticity simulations. Additionally, the effect of elastic deformation on the stress factor during the first few percent of deformation can be assessed.

\section{Conclusions}

From this study the following conclusions are drawn:

- Strain heterogeneity on the grain length scale plays a key role in the accurate prediction of hardening for textured, single-phase steels. The Alamel multi-scale homogenization model captures loading-dependent strain heterogeneities, enabling superior prediction of strain hardening compared to the iso-strain Taylor homogenization scheme.

- Texture development, which is obviously loading-dependent, plays an essential role in the prediction of differential hardening. It was found that for textured materials the strain heterogeneity is also loading-dependent and that it strengthens the differential hardening effect. In the macroscopic yield criterion, texture development has an impact on the yield function, i.e. texture evolution changes the yield locus shape.

- Prescribing the strain hardening through evolution of the critical resolved shear stress (CRSS) in the individual grains as a function of accumulated slip (termed microscopic hardening), leads to very similar stress predictions compared to directly defining a macroscopic flow stress that depends on the homogenized accumulated slip. In principle, the microscopic hardening assumption results in a loading-dependent flow stress in the macroscopic yield criterion.

- The Alamel model predicts more pronounced differential hardening for steel with stronger $\mathrm{v}$-fibre texture. This trend is in accordance with experimental observations.

- Possible reasons for the remaining discrepancy between prediction and observation of work hardening and differential hardening behaviour are included in the discussion; promising future developments include the consideration of loading-dependent evolution of grain substructure, as well as the incorporation of anisotropic crystal elasticity in full-field crystal plasticity models.

\section{Acknowledgements}

This research was carried out under the project numbers M41.10.08307b and M22.1.10394 in the framework of the Research Program of the Materials innovation institute M2i (www.m2i.nl). PE, JG, DR, AVB and PVH gratefully acknowledge the financial support from the Knowledge Platform M2Form, funded by IOF KU Leuven, and from the Belgian Federal Science Policy agency, contracts IAP7/19 and IAP7/21. 


\section{Appendix A: Procedure for calibration and stress-strain prediction}

\section{A.1. Macroscopic strain hardening definition}

For the macroscopic definition of strain hardening (section 2.3.1), specific stress-strain relations can be derived from (28) for different monotonic loading cases. In this paper in-plane uniaxial tension and ND-compression are considered:

Uniaxial tension: Let $\sigma_{11}$ be the tensile stress in a uniaxial tensile test with tensile direction 1 (at angle $\alpha$ to the RD) and in-plane transverse direction 2. The instantaneous anisotropic flow is expressed through the q-value: $q_{\alpha}=-A_{22} / A_{11}$. Equation (28) reduces to the following stress-strain relation:

$\sigma_{11}=\sqrt{2 / 3\left(1+q_{\alpha}^{2}+\left(1-q_{\alpha}\right)^{2}\right)} \bar{M} Y^{(M a)}$

ND compression: For ND compression loading, anisotropic flow is described by the equibiaxial q-value $q_{b}=-A_{R D} / A_{N D}$, in which $A_{R D}$ and $A_{N D}$ are the normal strain mode components along the $\mathrm{RD}$ and TD, respectively. In absolute value, the ND compressive stress $\sigma_{33}$ is then given by

$\left|\sigma_{33}\right|=\sqrt{2 / 3\left(1+q_{b}^{2}+\left(1-q_{b}\right)^{2}\right)} \bar{M} Y^{(M a)}$

It is reminded that $\bar{M}$ in (A.1)-(A.2) depends on the instantaneous strain mode and instantaneous state of material - cf. (15) and (19) for Taylor and Alamel, respectively.

For the macroscopic hardening law (27), the procedure for strain hardening parameter identification and consecutive stress-strain predictions comprises three stages:

(i) Polycrystalline plasticity simulation without strain hardening The equivalent stress $Y^{(M a)}$ of the polycrystal equals the CRSS that (by assumption) is identical for each constituting grain in any deformation stage. Therefore, the strain hardening is introduced conceptually at the macroscopic (homogenized) scale, and the polycrystalline plasticity model (FC Taylor or Alamel) is completely independent of strain hardening. This is realized in practice by setting the CRSS to unity for (i) all grains and (ii) all levels of deformation. If the (macro) level of deformation is represented by the von Mises equivalent strain $\varepsilon_{v M}=\int D_{v M} d t$, the polycrystalline plasticity model produces for any strain- or stressbased loading (including those of (A.1)-(A.2)) the following quantities independently from the hardening law (27):

- The volume-averaged slip rate $\bar{\Gamma}\left(\varepsilon_{v M}\right)$

- The (volume-) averaged Taylor factor $\bar{M}\left(\varepsilon_{v M}\right)$

- The macroscopic (or volume-averaged) strain mode $\mathbf{A}\left(\varepsilon_{v M}\right)$ For a stress-based loading such as uniaxial tension and ND-compression, the strain mode will evolve as a result of texture evolution. Consequently, also the q-value ( $q_{\alpha}$ in (A.1); $q_{b}$ in (A.2)) is a function of $\varepsilon_{v M}$. The evolution in q-value is one of the results produced by the algorithm for stress-defined deformation paths described in Appendix B. 
Note that the dependency of all 3 above quantities on the deformation level (described by $\varepsilon_{v M}$ ) is the direct result of change of material state during deformation. Neglecting changes in the material state, including any texture evolution, is thus equivalent to using the respective quantities at the onset of yielding (subscript ' 0 ') for all strain levels, i.e. $\bar{\Gamma}_{0}, \bar{M}_{0}$ and $\mathbf{A}_{\mathbf{0}}$.

(ii) Identification of the parameters $Y^{0}, \bar{\Gamma}^{0}$ and $N$ of (27) through stress-strain curve fitting The stress in uniaxial tension test along RD is given by (A.1) and (27), in which the accumulated volume-average slip $\bar{\Gamma}$ is found through integration: $\bar{\Gamma}\left(\varepsilon_{v M}\right)=\int \bar{\Gamma}\left(\varepsilon_{v M}\right) d \varepsilon_{v M}$. The only unknowns in this equation are thus the 3 hardening parameters, which are obtained through curve fitting with the experimental stress-strain curve.

(iii) The hardening law with identified parameters can afterwards be applied to the prediction of other loading cases, in casu uniaxial ND-compression (cf. (A.2)).

\section{A.2. Microscopic strain hardening definition}

For the microscopic strain hardening definition of section 2.3.2, the procedure is more straightforward, comprising firstly an identification step, followed by prediction of stress-strain curve(s):

(i) Identification of the parameters $\tau_{c}^{0}, \tau_{c}^{\text {sat }}$ and $c$ of (30) through stress-strain curve fitting The uniaxial tension stress is given in (A.1) together with (30), in which the accumulated volume-average slip $\bar{\Gamma}$ is found through integration: $\bar{\Gamma}\left(\varepsilon_{v M}\right)=\int \bar{\Gamma}\left(\varepsilon_{v M}\right) d \varepsilon_{v M}$. The only unknowns in this equation are thus the 3 hardening parameters, which are obtained through curve fitting with the experimental stress-strain curve. An essential difference with procedure A.1 is that texture evolution must be evaluated for every parameter set, while for the macroscopic hardening approach, the evaluation is effectively independent of the parameter set.

(ii) Secondly, the hardening law with identified parameters is applied to the prediction of other loadings, in casu uniaxial ND-compression. 


\section{Appendix B: Algorithm for stress-defined deformation paths}

This appendix presents an iterative procedure that allows one to analyse deformation paths defined by stress modes, even though a strain-rate driven crystal plasticity model is used. The crystal plasticity model is assumed to contain necessary microstructural state variables, such as texture and substructure. The model is then considered as a black-box implementing a purely plastic rateinsensitive material model. It must allow evaluating the homogenized macroscopic stress $\mathbf{S}_{H}$ as a response to the macroscopic strain rate $\mathbf{D}$, while keeping the state variables unmodified. An update of the state variables can be independently requested from the black-box.

The procedure exploits a transformation that allows converting rank-two tensor quantities of deviatoric nature into five-dimensional vectors. Detailed description of the transformation can be found in (Van Houtte and Van Bael, 2004). As a matter of convention, capital boldface letters (e.g. X) denote second-order deviatoric tensors and lowercase boldface letters (e.g. $\mathbf{x}$ ) stand for the corresponding vectors. There are two main reasons for preferring the five-dimensional vector representation over the direct tensor representation. Primarily, the conversion allows one to reduce dimensionality of the search space, since the constraint $\operatorname{tr}(\mathbf{X})=0$ is automatically satisfied by the five-dimensional vector representation. This becomes of importance since the algorithm presented in this appendix calculates all derivatives numerically. Therefore, the reduction in the space dimensionality allows decreasing the number of calls to the crystal plasticity model. Secondly, the underlying crystal plasticity model neglects elastic components of stress and strain rate, thus the homogenized stress is inherently deviatoric and the corresponding macroscopic strain rate has to satisfy volumetric incompressibility condition.

The fundamental part of the algorithm is to find the macroscopic plastic strain rate mode $\mathbf{a}^{*}$ that corresponds to the imposed deviatoric stress mode $\mathbf{b}^{*}$, where

$\mathbf{b} \stackrel{\text { def }}{=} \frac{\mathbf{s}}{\|\mathbf{S}\|}=\frac{\mathbf{S}}{\sqrt{\mathbf{S}: \mathbf{S}}}$

and $\mathbf{S}$ is the deviatoric part of a stress tensor defining the requested deformation path. This is achieved by solving an unconstrained optimization problem, in which the square norm of the vectorvalued residual function

$\mathbf{f}(\mathbf{a})=\mathbf{b}^{*}-\frac{\mathbf{s}_{H}(\mathbf{a})}{\left\|\mathbf{s}_{H}(\mathbf{a})\right\|}$

is minimized. The evaluation of the residual function involves a call to the underlying crystal plasticity to calculate homogenized stress $\mathbf{s}_{H}$. The search starts from an initial guess $\mathbf{a}_{0}$, typically chosen as $\mathbf{a}_{0}=\mathbf{b}^{*}$, which corresponds to the property of an isotropic von Mises plastic material. From a current point $\mathbf{a}_{\text {old }}$, the algorithm iteratively uses the trust-region approach (Conn et al, 2000):

$\min _{\mathbf{a} \in R^{5}}\left\|\mathbf{f}\left(\mathbf{a}_{\text {old }}\right)+\mathbf{J}\left(\mathbf{a}_{\text {old }}\right)\left(\mathbf{a}_{\text {new }}-\mathbf{a}_{\text {old }}\right)\right\|$ subject to $\left\|\mathbf{a}_{\text {new }}-\mathbf{a}_{\text {old }}\right\| \leq \Delta$

to find a new guess $\mathbf{a}_{\text {new }}=\mathbf{a}_{\text {old }}+\Delta \mathbf{a}$ that satisfies

$\min _{\mathbf{a} \in R^{5}}\left\|\mathbf{J}^{T}(\mathbf{a}) \mathbf{J}(\mathbf{a}) \Delta \mathbf{a}+\mathbf{J}(\mathbf{a}) \mathbf{f}(\mathbf{a})\right\|$

where $\Delta \mathbf{a}$ is the trial step, $\Delta$ is the size of the trial step and $\mathbf{J}$ is Jacobian matrix of the function $\mathbf{f}$. The search is terminated once any of the following criteria is fulfilled: (1) $\left\|\mathbf{f}\left(\mathbf{a}_{\text {new }}\right)\right\|$ is smaller than a pre- 
defined threshold $f_{\text {tol }}$, or (2) $\|\Delta \mathbf{a}\|$ is smaller than pre-defined minimal step size, or (3) the number of iterations exceeds its maximal value.

The evolution of the material state under the stress mode $\mathbf{b}^{*}$ can be calculated by means of Algorithm 1, which employs the search procedure outlined above. In principle, the algorithm splits the deformation path into smaller increments of strain for which the stress mode $\mathbf{b}^{*}$ is active, given the microstructural state of the material. Eventually the procedure returns a sequence of triplets $\left(\Delta \boldsymbol{\varepsilon}, \mathbf{A}^{*}, \mathbf{S}_{H}\right)$. Non-essential operations, such as reporting the history of microstructural state variables, are omitted for clarity.

Algorithm 1. Evolution of microstructural state variables subjected to the stress mode $\mathbf{b}^{*}$, calculated incrementally up to the norm of strain $\varepsilon_{\max }$. The size of increments is specified by the input parameter $\Delta \varepsilon$. The parameter $f_{\text {tol }}$ controls the convergence of the iterative search for $\mathbf{a}^{*}$. The procedure returns a sequence of triplets $\left(\Delta \boldsymbol{\varepsilon}, \mathbf{A}^{*}, \mathbf{S}_{H}\right)$.

Inputs: $\mathbf{b}^{*}, \varepsilon_{\max }, \Delta \varepsilon, f_{\text {tol }}$

Returns: A

1. Set $\boldsymbol{\varepsilon}_{\text {total }} \leftarrow 0$

2. Set A empty.

3. Find plastic strain rate $\mathbf{A}^{*}$ that corresponds to the superimposed $\mathbf{b}^{*}$ :

a. $\mathbf{a}^{*}=\operatorname{argmin}_{\mathbf{a} \in R^{5}}(\|\mathbf{f}(\mathbf{a})\|)$ given initial guess $\mathbf{a}_{0}=\mathbf{b}^{*}$

b. If $\|\mathbf{f}(\mathbf{a})\|>f_{\text {tol }}$ go to Step 10.

4. Convert $\mathbf{a}^{*}$ into $\mathbf{A}^{*}$

5. Calculate strain increment $\Delta \boldsymbol{\varepsilon}=\Delta \varepsilon \mathbf{A}^{*}$

6. Update state variables of the crystal plasticity model by applying $\Delta \varepsilon$ and calculate corresponding $\mathbf{S}_{H}$.

7. Append $\left(\Delta \varepsilon, \mathbf{A}^{*}, \mathbf{S}_{H}\right)$ to the sequence $\mathrm{A}$

8. Set $\varepsilon_{\text {total }} \leftarrow \varepsilon_{\text {total }}+\Delta \varepsilon$

9. If $\left\|\varepsilon_{\text {total }}\right\| \leq \varepsilon_{\max }$ go to Step 3 .

10. Stop 


\section{References}

An, Y.G., Vegter, H., 2005. Analytical and experimental study of frictional behavior in throughthickness compression test. Journal of Materials Processing Technology 160, 148-155.

An, Y.G., Vegter, H., Melzer, S., Romano Triguero, P., 2013. Evolution of the plastic anisotropy with straining and its implication on formability for sheet metals. Journal of Materials Processing Technology 213, 1419-1425.

Armstrong, P.J., Frederick, C.O., 1966. A mathematical representation of the multiaxial Bauschinger effect. Central Electricity Generating Board, Report RD/B/N 731.

Arul Kumar, M., Mahesh, S., Parameswaran, V., 2011. A 'stack' model of rate-independent polycrystals. International Journal of Plasticity 27, 962-981.

Asaro, R.J., Needleman, A., 1985. Overview No. 42: Texture Development and Strain Hardening in Rate Dependent Polycrystals. Acta Metallurgica, 923-963.

Bailey, J.E., Hirsch, P.B., 1960. The dislocation distribution, flow stress, and stored energy in coldworked polycrystalline silver. Philosophical Magazine 5, 485-497.

Banabic, D., Aretz, H., Comsa, D.S., Paraianu, L., 2005. An improved analytical description of orthotropy in metallic sheets. International Journal of Plasticity 21, 493-512.

Barlat, F., Aretz, H., Yoon, J.W., Karabin, M.E., Brem, J.C., Dick, R.E., 2005. Linear transfomation-based anisotropic yield functions. International Journal of Plasticity 21, 1009-1039.

Barlat, F., Brem, J.C., Yoon, J.W., Chung, K., Dick, R.E., Lege, D.J., Pourboghrat, F., Choi, S.-H., Chu, E., 2003. Plane stress yield function for aluminum alloy sheets - part 1: theory. International Journal of Plasticity 19, 1297-1319.

Barlat, F., Grácio, J.J., Lee, M.-G., Rauch, E.F., Vincze, G., 2011. An alternative to kinematic hardening in classical plasticity. International Journal of Plasticity 27, 1309-1327.

Barlat, F., Ha, J., Grácio, J.J., Lee, M.-G., Rauch, E.F., Vincze, G., 2013. Extension of homogeneous anisotropic hardening model to cross-loading with latent effects. International Journal of Plasticity 46, 130-142.

Bishop, J.F.W., Hill, R., 1951a. A theoretical derivation of the plastic properties of a polycrystalline face-centred metal. Philosophical Magazine 42, 1298.

Bishop, J.F.W., Hill, R., 1951b. A theory of the plastic distortion of a polycrystalline aggregate under combined stresses. Philosophical Magazine 42, 414.

Bunge, H.J., 1982. Texture Analysis in Materials Science: Mathematical Methods. Butterworth, London.

Cazacu, O., Barlat, F., 2004. A criterion for description of anisotropy and yield differential effects in pressure-insensitive metals. International Journal of Plasticity, 20(11), 2027-2045.

Cazacu, O., Plunkett, B., Barlat, F., 2006. Orthotropic yield criterion for hexagonal closed packed metals. International Journal of Plasticity, 22(7), 1171-1194.

Chaboche, J.L., 1986. Time-independent constitutive theories for cyclic plasticity. International Journal of Plasticity 2, 149-188.

Chung, K., Lee, M.-G., Kim, D., Kim, C., Wenner, M.L., Barlat, F., 2005. Spring-back evaluation of automotive sheets based on isotropic-kinematic hardening laws and non-quadratic anisotropic yield functions Part I: theory and formulation. International Journal of Plasticity 21, 861-882.

Conn, A. R., Gould N. I.M., Toint, P. L, 2000, Trust-region Methods. SIAM Society for Industrial \& Applied Mathematics, Englewood Cliffs, New Jersey, MPS-SIAM Series on Optimization edition 
Delannay, L., Melchior, M.A., Signorelli, J.W., Remacle, J.F., Kuwabara, T., 2009. Influence of grain shape on the planar anisotropy of rolled steel sheets - evaluation of three models. Computational Materials Science 45, 739-743.

Engler, O., Crumbach, M., Li, S., 2005. Alloy-dependent rolling texture simulation of aluminium alloys with a grain-interaction model. Acta Mater. 53, 2241-2257.

Evers, L.P., Parks, D.M., Brekelmans, W.A.M., Geers, M.G.D., 2002. Crystal plasticity model with enhanced hardening by geometrically necessary dislocation accumulation. J. Mech. Phys. Solids 50, 2403-2424.

Eyckens, P., Xie, Q., Sidor, J.J., Delannay, L., Van Bael, A., Kestens, L., Moerman, J., Vegter, H., Van Houtte, P., 2011. Validation of the texture-based ALAMEL and VPSC models by measured anisotropy of plastic yielding, in: Tewari, A., Suwas, S., Srivastava, D., Samajdar, I., Haldar, A. (Eds.), Materials Science Forum 702-703 (Proc. of ICOTOM 16). Trans Tech Publications, Mumbai, India, pp. 233-236.

Feigenbaum, H.P., Dugdale, J., Dafalias, Y.F., Kourousis, K.I., Plesek, J., 2012. Multiaxial ratcheting with advanced kinematic and directional distortional hardening rules. International Journal of Solids and Structures 49, 3063-3076.

Fernandes, J.V., Schmitt, J.H., 1983. Dislocation microstrucures in steel during deep drawing. Philosophical Magazine A 48, 841-870.

Freund, M., Shutov, A.V., Ihlemann, J., 2012. Simulation of distortional hardening by generalizing a uniaxial model of finite strain viscoplasticity. International Journal of Plasticity 36, 113-129.

Hansen, N., Juul Jensen, D., 2011. Deformed metals - structure, recrystallisation and strength. Materials Science and Technology 27, 1229-1240.

Hill, R., 1948. A theory of the yielding and plastic flow of anisotropic material. Proceedings of the Royal Society of London 193, 281-297.

Hill, R., Hecker, S.S., Stout, M.G., 1994. An investigation of plastic flow and differential work hardening in orthotropic brass tubes under fluid pressure and axial load. International Journal of Solids and Structures 31, 2999-3021.

Hill, R., Hutchinson, J.W., 1992. Differential hardening in sheet metal under biaxial loading: a theoretical framework. Journal of Applied Mechanics 59, S1-S9.

Kocks, U.F., 1998. Kinematics and Kinetics of Plasticity, in: Kocks, U.F., Tomé, C.N., Wenk, H.-R. (Eds.), Texture and Anisotropy: Preferred Orientations and their Effect on Materials Properties. Cambridge University Press, pp. 326-389.

Krabiell, A., Dahl, W., 1981. Zum Einfluß von Temperatur and Dehngeschwindigkeit auf die Streckgrenze von Baustählen unterschiedlicher Festigkeit. Archiv für das Eisenhüttenwesen 52, 429-436.

Kurtyka, T., Życzkowski, M., 1996. Evolution equations for distortional plastic hardening. International Journal of Plasticity 12, 191-213.

Kuwabara, T., Ikeda, S., Kuroda, K., 1998. Measurement and analysis of differential work hardening in cold-rolled steel sheet under biaxial tension. Journal of Materials Processing Technology 80-81, 517-523.

Lebensohn, R., Rollett, A., Suquet, P., 2011. Fast fourier transform-based modeling for the determination of micromechanical fields in polycrystals. JOM 63, 13-18.

Lebensohn, R.A., Tomé, C.N., 1993. A self-consistent anisotropic approach for the simulation of plastic deformation and texture development of polycrystals: application to zirconium alloys. Acta Metall. Mater., 2611-2624. 
Lee, B.J., Ahzi, S., Parks, D.M., 2002. Bicrystal-Based Modeling of Plasticity in FCC Metals. Journal of Engineering Materials and Technology 124, 27-40.

Ma, A., Roters, F., Raabe, D., 2006a. A dislocation density based constitutive model for crystal plasticity FEM including geometrically necessary dislocations. Acta Mater. 54, 2169-2179.

Ma, A., Roters, F., Raabe, D., 2006b. On the consideration of interactions between dislocations and grain boundaries in crystal plasticity finite element modeling - Theory, experiments, and simulations. Acta Mater. 54, 2181-2194.

Mahesh, S., 2010. A binary-tree based model for rate-independent polycrystals. International Journal of Plasticity 26, 42-64.

Mecking, H., Kocks, U.F., 1981. Kinetics of flow and strain hardening. Acta Metallurgica 29, 18651875.

Mulder, J., Vegter, H., 2010. Evolving anisotropic yield loci based on multiple hardening curves. In: Ceretti, E., Giardini, C. (Eds.), Proceedings of the $13^{\text {th }}$ International Esaform Conference on Material Forming, Brescia.

Peeters, B., Seefeldt, M., Teodosiu, C., Kalidindi, S.R., Van Houtte, P., Aernoudt, E., 2001. Workhardening/softening behaviour of b.c.c. polycrystals during changing strain paths: I. An integrated model based on substructure and texture evolution, and its prediction of the stress-strain behaviour of an IF steel during two-stage strain paths. Acta Mater. 49, 1607-1619.

Plunkett, B., Cazacu, O., Barlat, F., 2008. Orthotropic yield criteria for description of the anisotropy in tension and compression of sheet metals. International Journal of Plasticity 24, 847-866.

Prager, W., 1949. Recent developments in the mathematical theory of plasticity. Journal of Applied Physics 20, 235-241.

Roters, F., Eisenlohr, P., Hantcherli, L., Tjahjanto, D.D., Bieler, T.R., Raabe, D., 2010. Overview of constitutive laws, kinematics, homogenization and multiscale methods in crystal plasticity finiteelement modeling: Theory, experiments, applications. Acta Mater. 58, 1152-1211.

Taylor, G.I., 1938. Plastic strain in metals. J. Inst. Metals 62, 307-324.

Tjahjanto, D.D., Eisenlohr, P., Roters, F., 2010. A novel grain cluster-based homogenization scheme. Modelling and Simulation in Materials Science and Engineering 18, 015006.

Uenishi, A., Sugiura, N., Ikematsu, Y., Masaaki, S., Isogai, E., Hiwatashi, S., 2009. Work Hardening Behaviour and Dislocation Patterning at Large Strains in Sheet Steel, in: Higashida, K., Tsuji, N. (Eds.), Proceedings of the 2 nd International Symposium on Steel Science. The Iron and Steel Institute of Japan, Kyoto, Japan, pp. 57-62.

Van Houtte, P., 1988. A Comprehensive Mathematical Formulation of an Extended Taylor-Bishop-Hill Model Featuring Relaxed Constraints, the Renouard-Wintenberger Theory and a Strain Rate Sensitivity Model. Textures and Microstructures 8 \& 9, 313-350.

Van Houtte, P., Gawad, J., Eyckens, P., Van Bael, A., Samaey, G., Roose, D., 2011. A Full-field Strategy to Take Texture-induced Anisotropy into account during FE Simulations of Metal Forming Processes. JOM Journal of the Minerals, Metals and Materials Society 63, 37-43.

Van Houtte, P., Li, S., Seefeldt, M., Delannay, L., 2005. Deformation texture prediction: from the Taylor model to the advanced Lamel model. International Journal of Plasticity 21, 589-624.

Van Houtte, P., Van Bael, A., 2004, Convex plastic potentials of fourth and sixth rank for anisotropic materials. International Journal of Plasticity 20, 1505-1524.

Van Liempt, P., Sietsma, J., 2014. A physically based yield criterion for plastic deformation in metals. To be published. 
Van Riel, M., Van den Boogaard, A.H., 2007. Consistent plane stress-3D conversion of hardening models and yield criteria. In: Oñate, E., Owen, D.R.J., Suárez, B. (Eds.), Computational Plasticity: Fundamentals and Applications. CIMNE, Barcelona.

Vegter, H., Drent, P., Huétink, J., 1995. A planar isotropic yield criterion based on mechanical testing at multi-axial stress states. In: Shen, S.-F., Dawson, P.R. (Eds.), Simulation of Materials Processing: Theory, Methods and Applications. Balkema, Rotterdam, pp. 345-350.

Vegter, H., Van den Boogaard, A.H., 2006. A plane stress yield function for anisotropic sheet material by interpolation of biaxial stress states. International Journal of Plasticity 22, 557-580.

Von Mises, R., 1928. Mechanik der plastischen Formänderung von Kristallen. Zeitschrift für angewandte Mathematik und Mechanik 8, 161-185.

$\mathrm{Wu}, \mathrm{H} .-\mathrm{C} ., 2002$. Anisotropic plasticity for sheet metals using the concept of combined isotropickinematic hardening. International Journal of Plasticity 18, 1661-1682.

Xie, Q., Eyckens, P., Vegter, H., Moerman, J., Van Bael, A., Van Houtte, P., 2013. Polycrystalline model predictions of flow stress and textural hardening during monotonic deformation, in: Alves de Sousa, R., Valente, R. (Eds.), Key Engineering Materials (Proceedings of ESAFORM2013). Trans Tech Publications, Aveiro, Portugal, pp. 1157-1163.

Xie, Q., Van Bael, A., Sidor, J., Moerman, J., Van Houtte, P., 2014. A new cluster-type model for the simulation of textures of polycrystalline metals. Acta Mater. 69, 175-186.

Yoshida, F., Uemori, T., 2002. A model of large-strain cyclic plasticity describing the Bauschinger effect and workhardening stagnation. International Journal of Plasticity 18, 661-686. 